\title{
Turbulence Modeling Effects on the Prediction of Equilibrium States of Buoyant Shear Flows ${ }^{\star}$
}

\author{
C.Y. Zhao and R.M.C. So \\ Department of Mechanical Engineering, The Hong Kong Polytechnic University, \\ Hung Hom. Kowloon, Hong Kong, P.R. China
}

\section{T.B. Gatski}

Computational Modeling and Simulation Branch, NASA Langley Research Center, Hampton, VA 23681-2199, U.S.A.

Communicated by M.Y. Hussaini

Received 21 April 2000 and accepted 21 February 2001

\begin{abstract}
The effects of turbulence modeling on the prediction of equilibrium states of turbulent buoyant shear flows were investigated. The velocity field models used include a two-equation closure, a Reynoldsstress closure assuming two different pressure-strain models and three different dissipation rate tensor models. As for the thermal field closure models, two different pressure-scrambling models and nine different temperature variance dissipation rate $\left(\varepsilon_{\theta}\right)$ equations were considered. The emphasis of this paper is focused on the effects of the $\varepsilon_{t}$-equation. of the dissipation rate models, of the pressure-strain models and of the pressure-scrambling models on the prediction of the approach to equilibrium turbulence. Equilibrium turbulence is defined by the time rate of change of the scaled Reynolds stress anisotropic tensor and heat flux vector becoming zero. These conditions lead to the equilibrium state parameters, given by $\tilde{P} / \varepsilon, \tilde{P}_{H} / \varepsilon_{H}, R=\left(\bar{\theta}^{2} / 2 \varepsilon_{H}\right) /(k / \varepsilon), S k / \varepsilon$ and $G / \varepsilon$, becoming constant. Here, $\tilde{P}$ and $\tilde{P}_{H}$ are the production of turbulent kinetic energy $k$ and temperature variance $\overline{\theta^{2}}$, respectively, $\varepsilon$ and $\varepsilon_{\theta}$ are their respective dissipation rates, $R$ is the mixed time scale ratio, $G$ is the buoyant production of $k$ and $S$ is the mean shear gradient. Calculations show that the $\varepsilon_{H}$-equation has a significant effect on the prediction of the approach to equilibrium turbulence. For a particular $\varepsilon_{\sharp}$-equation, all velocity closure models considered give an equilibrium state if anisotropic dissipation is accounted for in one form or another in the dissipation rate tensor or in the $\varepsilon$-equation. It is further found that the models considered for the pressure-strain tensor and the pressure-scrambling vector have little or no effect on the prediction of the approach to equilibrium turbulence.
\end{abstract}

\section{Introduction}

Homogeneous turbulent flows played a central role in the modeling and analysis of complex inhomogeneous turbulent flows (Rogallo and Moin, 1984; Speziale, 1991). The reason is that these homogeneous flows provide a great deal of insight into key parameters characterizing turbulence in a simplified setting which quite

\footnotetext{
* The authors were supported through Grant No. Y-W18. The Hong Kong Polytechnic University, and Grant No. PolyU5154/99E. Research Grants Council, The Government of the HKSAR.
} 
often gives rise to closed-form solutions in the commonly used turbulence models. For turbulent shear flows without buoyancy, Lee and Reynolds (1985) and Rogers et al. (1986) have conducted direct numerical simulations (DNS) of such flows, and experimental investigations were carried out by Harris et al. (1977). Rohr 't al. (1988a) and Tavoularis and Karnik (1989). These investigations provided much-needed insight into the understanding of the evolution of homogeneous turbulence and its approach to equilibrium.

For turbulent buoyant shear flows, DNS have been performed by Gerz et al. (1989), Gerz and Schumann (1991) and Kaltenbach et al. (1994), while Piccirillo and van Atta (1997) and Rohr et al. (1988b) have experimentally studied the buoyancy effects on stratified turbulent shear flows. According to these investigations, stable stratification weakens isotropization. Therefore, the isotropic dissipation rate model is not adequate to describe the evolution of buoyant homogeneous turbulence. Recently, So et al. (1999) conducted a numerical simulation using different turbulence models and assessed the performance of certain anisotropic dissipation rate models. Their investigations revealed that there were difficulties still in the prediction of counter-gradient heat flux and the onset of internal gravity waves in buoyant shear flows. However, it should he pointed out that all the investigations mentioned above were only carried out for the short time period (or the near-field region of experiments), therefore, turbulence evolution for the long time was not reported due to experimental and numerical difficulties.

An important property of homogeneous turbulent shear flows is the appearance of the dynamic state parameters, which tend to approach equilibrium values in the long time limit. The equilibrium states provide an important benchmark in the calibration of closure models. For non-buoyant turbulent shear flows, Abid and Speziale (1993) calculated the equilibrium states for channel flows and homogeneous turbulent shear flows using Reynolds-stress closures. The fixed points associated with the equilibrium states for several homogeneous shear flows were determined from bifurcation diagrams (Speziale and Mac Giolla Mhuiris, 1989; Speziale. 1991: Speziale et al. 1996), and they were used to assess the stability of higher-order models and their ability to predict the correct equilibrium values. Recently, using representation theory. Jongen and Gatski ( 1998 ) also showed that equilibrium turbulence of homogeneous shear flows is defined by the state parianeters, $\tilde{P} / \varepsilon$ and $S k / \varepsilon$. Here, $\tilde{P}$ is the production of turbulent kinetic energy $k$ and $S$ is the mean shear rate of the mean flow. They further derived a general algebraic relation between the state parameters based solely on the form of the pressure-strain rate model. and without having to specify a modeled $\varepsilon$-equation for the dissipation rate of $k$. Their analysis gave the same equilibrium values as those predicted by the Reynolds-stress turbulence closures.

Tavoularis and Corrsin ( 1981 ) conjectured that equilibrium states also exist for buoyant shear flows, however, they take a longer time (or distance) to achieve. Due to the interaction between shear and buoyancy, the approach to equilibrium turbulence for buoyant shear flows is much more complicated. Up to now, even the parameters that characterize the equilibrium states are not known precisely and there was a lack of reliable experimental data on the equilibrium states of turbulent buoyant shear flows.

Numerical modeling of buoyant shear flows, even under the assumption of incompressibility, is quite a bit more complicated than pure shear flows. In addition to closure models for the Reynolds-stress equation, other models for the equations governing the transport of the Reynolds heat-flux and the associated dissipation rate $\left(\varepsilon_{\theta}\right)$ of the temperature variance $\left(\overline{H^{2}}\right)$ need to be invoked (Launder, 1978, 1989). Furthermore, buoyancy effects should also be modeled into the pressure-strain term based on the argument that buoyant forces enter into the Poisson equation for the fluctuating pressure. A modeled buoyancy term was also added to the $\varepsilon$-equation to account for buoyancy effects in the calculation of $\varepsilon$. It should be noted that this treatment of buoyancy effects on the turbulence field is rather incomplete. because the interaction between shear and buoyancy is highly nonlinear. This treatment also ignores the tight coupling between the Reynolds-stress equation and the heat-flux equation, because it simplifies the coupling between the different terms in these equations. Since the exact form of the modeled E-equation is not important in the prediction of the equilibrium states of pure shear flows, would the same conclusion be true for the $\varepsilon_{t}$-equation for buoyant shear flows. In view of this, there is a need to explore the ability of commonly used turbulence models to predict the equilibrium states of buoyant shear flows.

An analysis along the lines proposed by Jongen and Gatski (1998) was used to investigate the equilibrium state parameters of buoyant turbulent shear flows (Zhao et al., 2001). The requirements that $\mathrm{d} b_{i j} / \mathrm{d} t=0$ and $\mathrm{d} q_{i} / \mathrm{d} t=0$ lead to $\tilde{P} / \varepsilon, S k / \varepsilon, \tilde{P}_{H} / \varepsilon_{\theta,}, R=\left(\tilde{\theta}^{2} / 2 \varepsilon_{\theta}\right) /(k / \varepsilon)$ and $G / \varepsilon$ becoming constant at equilibrium. Here, $b_{i j}=\overline{\pi_{i} u_{j}} / 2 k-\delta_{i j} / 3$ is the scaled Reynolds stress anisotropic tensor. $q_{i}=\overline{u_{i} \theta} /\left(k \theta^{2}\right)^{1 / 2}$ is the scaled 
heat flux vector, $\overline{u_{i} u_{j}}$ is the kinematic Reynolds stress tensor, $\overline{u_{i} \theta}$ is the heat flux vector, $\tilde{P}_{\theta}$ is the production of the temperature variance $\overrightarrow{\theta^{2}}, \varepsilon_{\theta}$ is its dissipation rate, $R$ is the mixed time scale ratio and $G$ is the buoyant production of $k$. Therefore, the objective of this paper is to examine the effects of turbulence modeling on the prediction of these equilibrium states. It can be classified into the following tasks. The first task is to investigate the effect of the modeled s,-equation on the prediction of equilibrium states. A second task is to study the effect of modeling the pressure-strain tensor. The third task is to examine the effect of the model invoked for the pressure-scrambling vector. Finally, a fourth task is to investigate the influence of anisotropic dissipation modeling. Since this model affects the modeled $\varepsilon$-equation, the effect of this equation in the context of anisotropic dissipation modeling will be examined also. Two different turbulence closure schemes are used in this study, a $k-\varepsilon$ and a Reynolds-stress closure. The $k-\varepsilon$ closure is used to study in detail the effect of the modeled $\varepsilon_{H}$-equation, while the Reynolds-stress closure is used to examine the effects of pressure-strain models, pressure-scrambling models and dissipation rate tensor models.

\section{The Modeled Equations}

\section{Reynolds Averaged Equations}

The flow considered is a homogeneous buoyant turbulent shear flow where the Boussinesq approximation is assumed valid. According to the studies of Sommer and co-workers $(1995,1997)$, two-equation heat flux models are not appropriate for buoyant shear flows, because they failed to predict the onset of countergradient heat flux and the presence of internal gravity waves correctly. Therefore, a Reynolds heat-flux model has to be assumed instead. Invoking the Boussinesq approximation, the incompressible Reynolds-stress and heat-flux modeled transport equations can be written for a homogeneous shear flow as

$$
\begin{aligned}
& \frac{\mathrm{d} \overline{u_{i} u_{j}}}{\mathrm{~d} t}=P_{i j}+\Pi_{i j}-\varepsilon_{i j}+G_{i j}, \\
& \frac{\mathrm{d} \overline{u_{i} \theta}}{\mathrm{d} t}=-\overline{u_{i} u_{j}} \frac{\partial(-)}{\partial x_{j}}-\overline{u_{i} \theta} \frac{\partial U_{i}}{\partial x_{j}}+\Phi_{H i}+G_{H_{i}} .
\end{aligned}
$$

where $U_{i}$ and $u_{i}$ are the $i$ th component of the mean and fluctuating velocity, $\Theta$ and $\theta$ are the mean and fluctuating temperature, $t$ is time, $x_{i}$ is the $i$ th component of the coordinate, $P_{i j}=-\bar{u}_{i} u_{k}\left(\partial U_{j} / \partial x_{k}\right)-$ $\overline{u_{i} u_{k}}\left(\partial U_{i} / \partial x_{k}\right)$ is the shear stress production tensor, $G_{i j}=-\beta g_{i} \overline{u_{j} \theta}-\beta g_{j} \overline{u_{i} \theta}$ is the buoyant production of the Reynolds stresses and $G_{H i}=-\beta g_{i} \overline{\theta^{2}}$ is the buoyant production of the heat fluxes. Here, $\beta$ is the coefficient of thermal expansion of the fluid and $g_{i}$ is the gravitational vector. The pressure-strain tensor $\Pi_{i j}$ and the dissipation rate tensor $\varepsilon_{i j}$ in (1) need modeling. The $\Phi_{\theta i}$ term includes both the pressure-scrambling vector and the molecular dissipation of the heat fluxes. Its writing was suggested by Launder (1978). Therefore, its modeling should include the effect of molecular dissipation on the heat fluxes. These models are briefly discussed below.

\section{Pressure-Strain Models}

For buoyant turbulent flows, the term $\Pi_{i j}$ can be represented by three parts; they are the slow part $\Pi_{i j}^{(S)}$. the rapid part $\Pi_{i j}^{(\mathrm{R})}$ and the buoyant part $\Pi_{i j}^{(\mathrm{B})}$. The slow and rapid parts can be modeled as in non-buoyant flows and the buoyant part can be modeled by relating it to the generation/destruction of the Reynolds stresses by buoyancy (Launder, 1978). Two different models for $\Pi_{i j}^{(S)}+\Pi_{i j}^{(\mathrm{R})}$ are investigated; they are the linear IP model of Launder et al. (1975) and the nonlinear SSG model of Speziale et al. (1991). These models plus the buoyant model, $\Pi_{i j}^{(\mathrm{B})}=C_{6}\left(G_{i j}-2 G \delta_{i j} / 3\right)$, can be written as follows. The complete expression for 
$\Pi_{i j}$, invoking the IP model for $\Pi_{i j}^{(\mathrm{S})}+\Pi_{i j}^{(\mathrm{R})}$, is given by

$$
\begin{aligned}
\Pi_{i j}= & -C_{1} \varepsilon b_{i j}+C_{2} k S_{i j}+C_{3} k\left(b_{i k} S_{j k}+b_{j k} S_{i k}-\frac{2}{3} b_{m n} S_{m n} \delta_{i j}\right) \\
& +C_{4} k\left(b_{i k} W_{j k}+b_{j k} W_{i k}\right)-C_{6}\left(G_{i j}-\frac{2}{3} G \delta_{i j}\right) .
\end{aligned}
$$

where $C_{1}=3.6, C_{2}=0.8, C_{3}=1.2, C_{4}=1.2, C_{6}=0.3$ and $G=G_{i i} / 2$ is the buoyant production of $k$. The corresponding expression for $\Pi_{i j}$, invoking the SSG model for $\Pi_{i j}^{(S)}+\Pi_{i j}^{(\mathrm{R})}$, is

$$
\begin{aligned}
\Pi_{i j}= & -\left(C_{1}+C_{1}^{*} \tilde{P}\right) \varepsilon b_{i j}+C_{2} k S_{i j}+C_{3} k\left(b_{i k} S_{j k}+b_{j k} S_{i k}-\frac{2}{3} b_{m n} S_{m n} \delta_{i j}\right) \\
& -C_{4} k\left(b_{i k} W_{j k}-b_{j k} W_{i k}\right)+C_{5} \varepsilon\left(b_{i k} b_{k j}-\frac{1}{3} b_{m n} b_{n m} \delta_{i j}\right)-C_{6}\left(G_{i j}-\frac{2}{3} G \delta_{i j}\right) .
\end{aligned}
$$

The model constants are specified as $C_{1}=3.4, C_{2}=0.36, C_{3}=1.25, C_{4}=0.40, C_{5}=4.2, C_{1}^{*}=1.8$ and $C_{6}=0.3$. In these equations the kinematic strain rate $\left(S_{i j}\right)$ and rotation rate $\left(W_{i j}\right)$ tensors are defined as $S_{i j}=\left(\partial U_{i} / \partial x_{j}+\partial U_{j} / \partial x_{i}\right) / 2$ and $W_{i j}=\left(\partial U_{i} / \partial x_{j}-\partial U_{j} / \partial x_{i}\right) / 2$, respectively.

\section{Dissipation Rate Models}

The dissipation rate tensor $\varepsilon_{i j}$ can be modeled using three different approaches. They are the isotropic model (Kolmogorov, 1941) and two different algebraic models (Hallback et al., 1990; Speziale and Gatski, 1997). These models are given here for reference without derivation. The Kolmogorov (1941) isotropic model is given by

$$
\varepsilon_{i j}=\frac{2}{3} \varepsilon \delta_{i j}
$$

while the anisotropic algebraic models of Hallback et al. (1990) and Speziale and Gatski (1997), designated as HGJ and SG, respectively, can be written in the general form as

$$
\varepsilon_{i j}=\frac{2}{3} \varepsilon \delta_{i j}+2 \varepsilon d_{i j} .
$$

In the HGJ model, $d_{i j}$ is expressed as a series of $b_{i j}$, while in the SG model, $d_{i j}$ is expressed in terms of $S_{i j}$ and $W_{i j}$. For the HGJ model, the expression for $d_{i j}$ is given by

$$
d_{i j}=\frac{1}{2}\left[1+\alpha^{\prime}\left(\frac{1}{2} \Pi-\frac{2}{3}\right)\right] b_{i j}-\alpha^{\prime}\left(b_{i k} b_{k j}-\frac{1}{3} \Pi \delta_{i j}\right),
$$

where $\alpha^{\prime}=\frac{3}{4}$ has been assumed. The SG modeled equation for $d_{i j}$ is given by

$$
\begin{aligned}
& d_{i j}=-2 C_{\mu \varepsilon} {\left[\frac{k}{\varepsilon} S_{i j}+\left\{\frac{A_{1}}{C_{\varepsilon 5}+\tilde{P} / \varepsilon-1}\right\} \frac{k^{2}}{\varepsilon^{2}}\left(S_{i k} W_{k j}+S_{j k} W_{k i}\right)\right] } \\
&+2 C_{\mu \varepsilon}\left\{\frac{A_{2}}{C_{\varepsilon 5}+\tilde{P} / \varepsilon-1}\right\} \frac{k^{2}}{\varepsilon^{2}}\left(S_{i k} S_{k j}-\frac{1}{3} S_{k l} S_{k l} \delta_{i j}\right) .
\end{aligned}
$$

where $\alpha_{3}=0.6 . A_{1}=\frac{7}{11} \alpha_{3}+\frac{1}{11}$ and $A_{2}=\frac{30}{11} \alpha_{3}-\frac{2}{11}$. The coefficient $C_{\mu \varepsilon}$ is defined as

$$
C_{\mu \varepsilon}=\frac{1}{15\left(C_{\varepsilon 5}+\tilde{P} / \varepsilon-1\right)}\left[1+2\left(\frac{A_{1}}{C_{\varepsilon 5}+\tilde{P} / \varepsilon-1}\right)^{2} \xi_{1}^{2}-\frac{2}{3}\left(\frac{A_{3}}{C_{\varepsilon 5}+\tilde{P} / \varepsilon-1}\right)^{2} \eta_{1}^{2}\right] .
$$

Two new constants are introduced and they are $C_{\varepsilon 5}=5.80, A_{3}=\frac{15}{11} \alpha_{3}-\frac{1}{11}$. The dimensionless strain invariants are defined by $\eta_{1}=\left(S_{i j} S_{i j}\right)^{1 / 2}(k / \varepsilon)$ and $\xi_{1}=\left(W_{i j} W_{i j}\right)^{1 / 2}(k / \varepsilon)$. 
The $\varepsilon_{i j}$ model requires knowledge of $\varepsilon$ for closure. For the HGJ model, the $\varepsilon$-equation solved is the conventional equation, which after modification for buoyant flows becomes

$$
\frac{\mathrm{d} \varepsilon}{\mathrm{d} t}=C_{\varepsilon 1} \frac{\varepsilon}{k} \tilde{P}-C_{\varepsilon: 2} \frac{\varepsilon^{2}}{k}-C_{\varepsilon 3} \frac{\varepsilon}{k} \beta g_{i} \overline{u_{i} \theta}
$$

where $\tilde{P}$ is one-half the trace of $P_{i j}$. The model constants take on the following values: $C_{\varepsilon 1}=1.44$, $C_{\varepsilon 2}=1.83$ and $C_{\varepsilon 3}=1.44$. Note that (10) does not include the effect of $d_{i j}$, whose influence only contributes to the calculation of $\varepsilon_{i j}$ through (6). This, however, is not the case for the SG model, and the $\varepsilon$-equation solved is modified to give

$$
\frac{\mathrm{d} \varepsilon}{\mathrm{d} t}=C_{\varepsilon 1} \frac{\varepsilon}{k} \tilde{P}-2(1+\gamma) \varepsilon d_{i j} S_{i j}-C_{\varepsilon 2} \frac{\varepsilon^{2}}{k}-C_{\varepsilon} \frac{\varepsilon}{k} \beta g_{i} \overline{u_{i} \theta}
$$

where $\gamma=\frac{3}{4}\left(\frac{14}{11} \alpha_{3}-\frac{16}{13}\right)$ and the constants take on values $C_{\varepsilon 1}=1.0, C_{\varepsilon 2}=1.83$ and $C_{\varepsilon 3}=1.44$. In this equation the effects of $d_{i j}$ are accounted for through the term involving both $d_{i j}$ and $S_{i j}$. Thus, the anisotropic effects of $\varepsilon_{i j}$ are given by $(6)$ as well as accounted for in the determination of $\varepsilon$. This is a major difference between the HGJ and SG models.

\section{Heat Flux Models}

Just like $\Pi_{i j}$, the term $\Phi_{H i}$ can again be partitioned into three parts, a slow part $\Phi_{H i}^{(\mathrm{S})}$, a rapid part $\Phi_{t i i}^{(\mathrm{R})}$, and a buoyant part $\Phi_{H i}^{(\mathrm{B})}$. Usually, the Monin (1965) model is invoked for $\Phi_{H i}^{(\mathrm{S})}$. However. Lumley and KhajehNouri (1974) proposed a quadratic modification to Monin's (1965) model to account for the anisotropy of the heat flux. Their proposal leads to $\Phi_{\theta i}^{(\mathrm{S})}=-C_{1 H} \varepsilon \overline{u_{i} \theta} / k-2 C_{1 \theta}^{\prime} \varepsilon b_{i j} \overline{u_{j} \theta} / k$. The rapid part is usually modeled by $\Phi_{\theta i}^{(\mathrm{R})}=b_{k i}^{m}\left(\partial U_{k} / \partial x_{m}\right)$ with different models proposed for $b_{k i}^{m}$. Recently, Craft and Launder (1989) derived a fairly complete expression for $b_{k i}^{m}$ based on the realizability condition in terms of Schwarz's inequality for the heat fluxes and by imposing additional conditions. Their expression involves quadratic terms of $b_{i j}$ in addition to terms linear in the heat flux. If all quadratic terms involving turbulent stresses and heat fluxes are neglected and the model $\Phi_{\theta i}^{(\mathrm{B})}=C_{5 H} g_{i} \beta \theta^{2}$ (Launder, 1978) is invoked for the buoyant part. the complete model for $\Phi_{t i}$ can be written as (So and Speziale, 1999)

$$
\Phi_{\theta i}=-C_{1 H} \frac{\varepsilon}{k} \overline{u_{i} \theta}+C_{2 \theta} \overline{u_{j} \theta} \frac{\partial U_{i}}{\partial x_{j}}+C_{3 \theta} \overline{u_{j} \theta} \frac{\partial U_{j}}{\partial x_{i}}+C_{5 \theta} g_{i} \beta \overline{\theta^{2}} .
$$

where $C_{5 \theta}=C_{2 \theta}$ is recommended by Launder (1978). If the term involving $C_{3 \theta}$ is set to zero as suggested by Launder (1978), the other constants would take on the following values, $C_{1 H}=3.28$ and $C_{2 H}=0.40$. On the other hand, including the $C_{3 H}$ term leads to the recommended constants $C_{1 H}=3.28, C_{2 H}=0.80$ and $C_{3 \theta}=-0.20$.

The linear model given in (12) is by no means unique. For example, Shabany and Durbin (1997) suggested a slightly different linear model for $\Phi_{\theta i}^{(R)}$. Their proposal consists of three terms, the $C_{2 \theta}$ and $C_{3 \theta}$ term plus a term involving the mean temperature gradient. $C_{4 i} \overline{u_{i} u_{j}}\left(\partial \Theta / \partial x_{j}\right)$. However, in their calculations, they have set $C_{4 \theta}=0$. Therefore, in reality, their suggested linear model for $\Phi_{\theta i}^{(\mathrm{R})}$ is the same as that given in (12). A comparison of the effects of the different terms in (12) including a $C_{4 \theta}$ term on heat flux modeling has been attempted by Wikstrom et al. (2000). Their comparisons were made with DNS data, whenever available, obtained for a homogeneous shear flow, a plane channel flow and a heated cylinder wake. In addition, they have also considered models where the $C_{1 H}$ term was modified by the time scale ratio $R$. Their results showed that if the $C_{4 \theta}$ term is included in (12), then $C_{2 \theta}$ and $C_{3 \theta}$ have to be set equal to zero. Otherwise, the model performance would deteriorate significantly. A model that performs well for all test cases was one given by modifying $C_{1 H}$ with $R$ and setting all other constants to zero. Other models perform differently for the test cases considered.

As a first attempt, the present study focuses on (12) only. It should be pointed out that the proposed model includes the effect of molecular dissipation on the heat fluxes and is consistent with those used by other researchers. All calculations reported in this paper, unless specified explicitly, were carried out assuming $C_{3 H}$ to be zero. The effect of the $C_{3 \theta}$ term on the prediction of equilibrium states is investigated separately. 


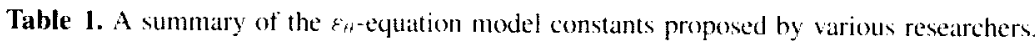

\begin{tabular}{|c|c|c|c|c|c|}
\hline Modeled $\varepsilon_{i}$-equation & $C_{d 1}$ & 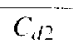 & $C_{d i}$ & $C_{i t}$ & $C_{d 5}$ \\
\hline NLL. (Newman et al. 1981 ) & 2.00 & 0 & 0 & 2.02 & 0.88 \\
\hline EL (Elghobashi and Launder. 1983) & 1.80 & 0 & 0 & 2.20 & 0.80 \\
\hline GJK (Gibson er al., 1987) & 0 & 1.70 & 1.40 & 2.00 & 1.80 \\
\hline AY (Yoshizawa, 1988) & 1.20 & 0 & 0.52 & 1.20 & 0.52 \\
\hline JM (Jones and Musonge, 1988) & 0 & 1.70 & 1.40 & 2.00 & 0.52 \\
\hline NK (Nagano and Kim, 1988) & 1.80 & ) & 0.72 & 2.20 & 0.80 \\
\hline CL (Craft and Lalunder, 1989) & () & $1.30)$ & 0 & 2.00 & 0.92 \\
\hline YNT (Youssef of al. 1992) & 1.70 & ) & 0.64 & $2 .(H)$ & 0.90 \\
\hline SS\% (Sommer et al. 1997) & ) & 1.20 & 0.72 & 2.20 & 0.80 \\
\hline
\end{tabular}

In view of the temperature coupling between (1) and (2), their closure requires knowledge of the variation of $\overline{\theta^{2}}$. This can be obtained by solving the modeled $\overline{\theta^{2}}$ and $\varepsilon_{\theta}$ transport equations. Nine different sets of modeled equations are summarized in So and Speziale (1999) and they can be written in a general form as

$$
\begin{aligned}
& \frac{\mathrm{d} \overline{\theta^{2}}}{\mathrm{~d} t}=2 \tilde{P}_{t \prime}-2 \varepsilon_{H} . \\
& \frac{\mathrm{d} \varepsilon_{\theta t}}{\mathrm{~d} t}=C_{d l} \frac{\varepsilon_{H}}{\overline{\theta^{2}}} \tilde{P}_{\theta}+C_{d 2} \frac{\varepsilon}{k} \tilde{P}_{H}+C_{d l 3} \frac{\varepsilon_{\theta}}{k} \tilde{P}-C_{d 4} \frac{\varepsilon_{H}}{\overline{\theta^{2}}} \varepsilon_{H}-C_{d 5} \frac{\varepsilon}{k} \varepsilon_{H} .
\end{aligned}
$$

where $\tilde{P}_{H}=-\overline{u_{i} \theta}\left(\partial(-) / \partial x_{i}\right)$. The different model constants adopted for pure and wall shear flows in the nine sets of modeled $\varepsilon_{H}$-equation are summarized in Table 1, where abbreviations (shown in Table 1) are used to denote the different models. These models are not completely independent of each other. For example, NLL and EL belong to one group. GJK, JM and SSZ another, AY, NK and YNT a third and CL is the fourth group. Members within a group only differ in the values of the constants chosen. In view of this, only one representative member of each group needs to be investigated.

\section{Numerical Solution}

Different combinations of the velocity governing equations given above will give rise to different Reynoldsstress closure models. For the sake of clarity, the following abbreviation are adopted to designate the various Reynolds-stress closures used for the calculations of turbulent buoyant shear flows. The 2-Eq designation is used to denote a two-equation model, which solves the trace of $(1)$ and (10), the IP designation means adopting (3). (5) and (10), while SSG means the use of (4), (5) and (10) in the equation set. It should be noted that, in the 2-Eq model, $\tilde{P}$ is approximated by $C_{\mu}\left(k^{2} / \varepsilon\right) S_{i j} S_{i j}$ with $C_{\mu}=0.09$ and the constants associated with $(10)$ are given by $C_{\varepsilon 1}=1.50, C_{\varepsilon 2}=1.90$ and $C_{k, 3}=1.50$. Other designations used are SSG $/ \mathrm{HGJ}$ and SSG/SG. They signify the solution of the equation set (4), (6), (7) and (10) and the set (4), (6), (8), (9) and (11), respectively. Of course, in all these closures, (1), (2) and (12)-(14) are also solved simultaneously. These closures differ in the modeling of the pressure-strain and dissipation rate tensors, and the pressurescrambling vector. Comparisons of these models can. therefore, shed light on their ability or inability to predict equilibrium states of turbulent buoyant shear flows.

The equations constitute a set of coupled initial value problems. They are solved using a Bulirsch-Stoer method (see Press et al., 1986) with variable step size in order to achieve the desired accuracy with as few steps as possible. Calculations were carried out with the dimensionless equations. The physical equations were normalized assuming the length scale to be given by $L$, the time scale by $1 / S$, the velocity scale by $\Delta U=S L$ and the temperature scale by $\Delta(-)=S L$. where $S=\mathrm{d} U / \mathrm{d} z$. Therefore, the dimensionless time is given by $\tau=S t$. It should be pointed out that even though the governing equations are not dependent on the molecular Prandtl number, $\operatorname{Pr}$, the initial conditions are. The $\operatorname{Pr}$ dependence comes in through the specification of the initial $\varepsilon_{\theta}$, which according to its definition involves the molecular thermal diffusivity. In view of this. Pr only affects the turbulence calculations in the initial stage of development but not in the long time period. The initial conditions are different for different flow cases. Since the present study examines the 
Table 2. The calculated values of $(\tilde{P} / \varepsilon)_{x}$ and $(S k / \varepsilon)_{\chi}$ for homogeneous shear flows.

\begin{tabular}{cccccc}
\hline & & \multicolumn{4}{c}{ Reynolds-stress closures } \\
& $\begin{array}{c}2 \text {-Eq } \\
\text { closure }\end{array}$ & IP & SSG & SSG/HGJ & SSG/SG \\
\hline$(\tilde{P} / \varepsilon)_{x}$ & 1.886 & 1.800 & 1.886 & 1.886 & 1.901 \\
$(S k / \varepsilon)_{2}$ & 4.578 & 4.927 & 5.760 & 5.938 & 5.983 \\
\hline
\end{tabular}

approach to equilibrium turbulence of the cases considered by So ef al. (1999), the same initial conditions specified there are used here. For most cases, unless specifically stated, $\operatorname{Pr}=5$ is assumed.

\section{Effect of Reynolds Stress Closures on the Equilibrium States of Pure Shear Flows}

The performance of different turbulence models in the predictions of homogeneous shear flows. homogeneous buoyant flows and homogeneous buoyant shear flows has been critically examined (So et al., 1999) and the calculations were evaluated against DNS and experimental data. However, the approach to equilibrium turbulence and the ability of these closure models to predict equilibrium states have not been examined. This paper proposes to examine the equilibrium states of stably stratified shear flows. Therefore, before proceeding to analyze buoyant shear flows, the effect of the different Reynolds stress models on the prediction of equilibrium states for pure shear flows has to be examined first.

For homogeneous shear flows, the approach to equilibrium turbulence is signified by $\mathrm{d} b_{i j} / \mathrm{d} t=0$. This leads to $\tilde{P} / \varepsilon$ and $S k / \varepsilon$ becoming constant as time goes to infinity (Jongen and Gatski, 1998). It may take a long time to reach this state. but large eddy simulation results (Rogers et al., 1986) show that such a state is achieved at equilibrium. Based on this condition and the governing $k$ and $\varepsilon$ equations, the asymptotic values for $\tilde{P} / \varepsilon$ and $S k / \varepsilon$ can be determined. According to Jongen and Gatski (1998), the condition $\mathrm{d}(S k / \varepsilon) / \mathrm{d} t=0$ is valid at equilibrium. Then it follows that

$$
\frac{\mathrm{d}(S k / \varepsilon)}{\mathrm{d} t}=\frac{S \mathrm{~d} k}{\varepsilon} \frac{S k}{\mathrm{~d} t}-\frac{\mathrm{d} \varepsilon}{\varepsilon^{2}} \frac{\mathrm{d} t}{\mathrm{~d} t} .
$$

Substituting the trace of $(1)$ and $(10)$ into (15) yields the asymptote, $(\tilde{P} / \varepsilon)_{\infty}=\left(C_{k 2}-1\right) /\left(C_{\varepsilon 1}-1\right)$. An explicit expression for $S k / \varepsilon$ is possible under the assumption of $\tilde{P}=C_{\mu}\left(k^{2} / \varepsilon\right) S^{2}$. This leads to $(S k / \varepsilon)_{\chi}=$ $\left[\left(C_{\varepsilon 2}-1\right) / C_{\mu}\left(C_{\varepsilon 1}-1\right)\right]^{1 / 2}$. Using the values of the constants specified for $(10),(\tilde{P} / \varepsilon)_{\infty}=1.886$ and $(S k / \varepsilon)_{\infty}=4.578$ is deduced. This shows that production does not balance dissipation as equilibrium is approached. Instead, at equilibrium. $\tilde{P}$ settles to a higher value than $\varepsilon$.

The next task is to evaluate the asymptotic values determined from the different Reynolds-stress closures. Only the model calculations from SSG and IP are considered, but they include the use of three different $\varepsilon_{i j}$ models, i.e. those given by (5), (6) and (7), and (6), (8) and (9), respectively. The calculations are compared with the 2-Eq model results in Table 2. For the present calculations, the values of $C_{\varepsilon 1}$ and $C_{\varepsilon 2}$ adopted for the 2-Eq. IP and SSG closures do not differ by much. Consequently, the asymptotic values of $\tilde{P} / \varepsilon$ determined from the model calculations cluster around 1.80. The correctness of these asymptotes for homogeneous shear flows remains to be verified. However, it should be pointed out that the experimental measurements of Tavoularis and Karnik (1989) indicate that $(S k / \varepsilon)_{\infty}$ should fall between 5.0 and 6.0 and Speziale and Gatski (1997) also obtained a value for $(S k / \varepsilon)_{\infty}$ close to 6.0. Based on this data, it appears that both the IP and the SSG results are fairly reasonable. irrespective of the dissipation rate tensor model assumed. All closure models considered predict a $(\tilde{P} / \varepsilon)_{\infty}$ value close to 1.9 instead of 1 as a production-balance-dissipation model would imply.

\section{Equilibrium States of Buoyant Shear Flows}

For buoyant shear flows, the approach to equilibrium is further complicated by the presence of the thermal field. What additional parameters besides $b_{i j}, \tilde{P} / \varepsilon$ and $S k / \varepsilon$ should be used to characterize the equilibrium states? The DNS data of Gerz et al. (1989) and the experimental measurements of Sirivat and Warhaft (1983) 

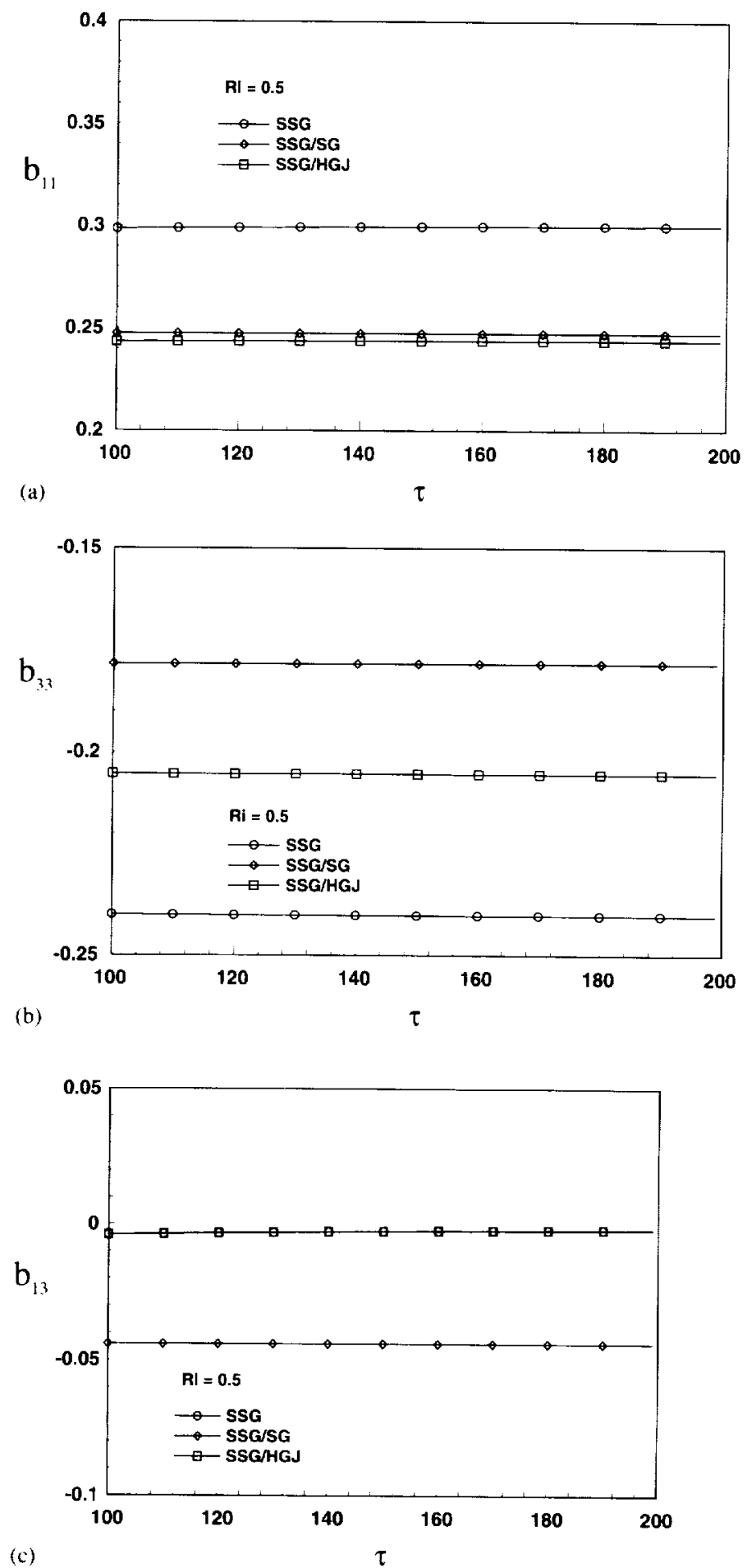

Figure 1. Variation of the components of $b_{i j}$ with time assuming the $\mathrm{JM}$ model.

could be interpreted to imply that the time rate of change of $b_{i j}$ and $q_{i}$ should be zero as equilibrium is approached. Furthermore, as the study of Zhao et al. (2001) showed, equilibrium buoyant turbulence as defined by $\mathrm{d} b_{i j} / \mathrm{d} t=0$ and $\mathrm{d} q_{i} / \mathrm{d} t=0$ will give rise to the state parameters $\tilde{P} / \varepsilon, S k / \varepsilon, \tilde{P}_{\theta} / \varepsilon_{\theta}, R$ and $G / \varepsilon$ becoming constant. Therefore, this section investigates the approach to equilibrium turbulence as predicted by the different closure models. In anticipation of results to be presented later, only the velocity model calcula- 
tions of IP, SSG, SSG/HGJ and SSG/SG, assuming an $\varepsilon_{H}$-equation model given either by JM or NK for a given Richardson number, $R i=\beta g(\mathrm{~d} \Theta / \mathrm{d} z) /(\mathrm{d} U / \mathrm{d} z)^{2}=0.5$, and $P r=5$, are shown in Figures $1-8$. This calculated case is identical to the DNS data of Gerz et al. (1989). The conclusions drawn from NK are also applicable to NLL and CL. Therefore, results obtained using NLL and CL and other members of the same group are not shown for the sake of brevity.

The time variation of the components of $b_{i j}$ and $q_{i}$ calculated using a number of velocity models and the JM model for the $\varepsilon_{H}$-equation are plotted in Figures $I$ and 2, respectively. Only the results for the SSG, SSG/HGJ and SSG/SG are shown. It is obvious that the Reynolds stresses (Figure 1) have reached their respective constants as early as $\tau=100$. As for the Reynolds heat fluxes, the stream component $q 1$ (Figure 2(a)) behaves like the Reynolds stresses, but the normal component $q 3$ (Figure 2(b)) reaches a constant value at $\tau \approx 160$. Thus, equilibrium is achieved only after $\tau=160$. Once $\mathrm{d} b_{i j} / \mathrm{d} t=0$ and $\mathrm{d} q_{i} / \mathrm{d} t=0$ have been achieved, $R$ also reaches a constant value (Figure 3 ). The constant value reached is different for different velocity models. This is not unexpected, because the equilibrium values of $(\tilde{P} / \varepsilon)_{\infty}$ and $(S k / \varepsilon)_{\infty}$ are different for different velocity models for pure shear flows as indicated in Table 1. However, constant $R$ does not necessarily imply that $\mathrm{d}(S k / \varepsilon) / \mathrm{d} t$ will go to zero also. It follows from the definition of $R$ that when $R$ and $S k / \varepsilon$ are becoming constant, the following relation can be deduced:

$$
\frac{\mathrm{d}(k / \varepsilon)}{\mathrm{d} t}=\frac{\mathrm{d}\left(\overline{\theta^{2}} / \varepsilon_{H}\right)}{\mathrm{d} t}=0
$$
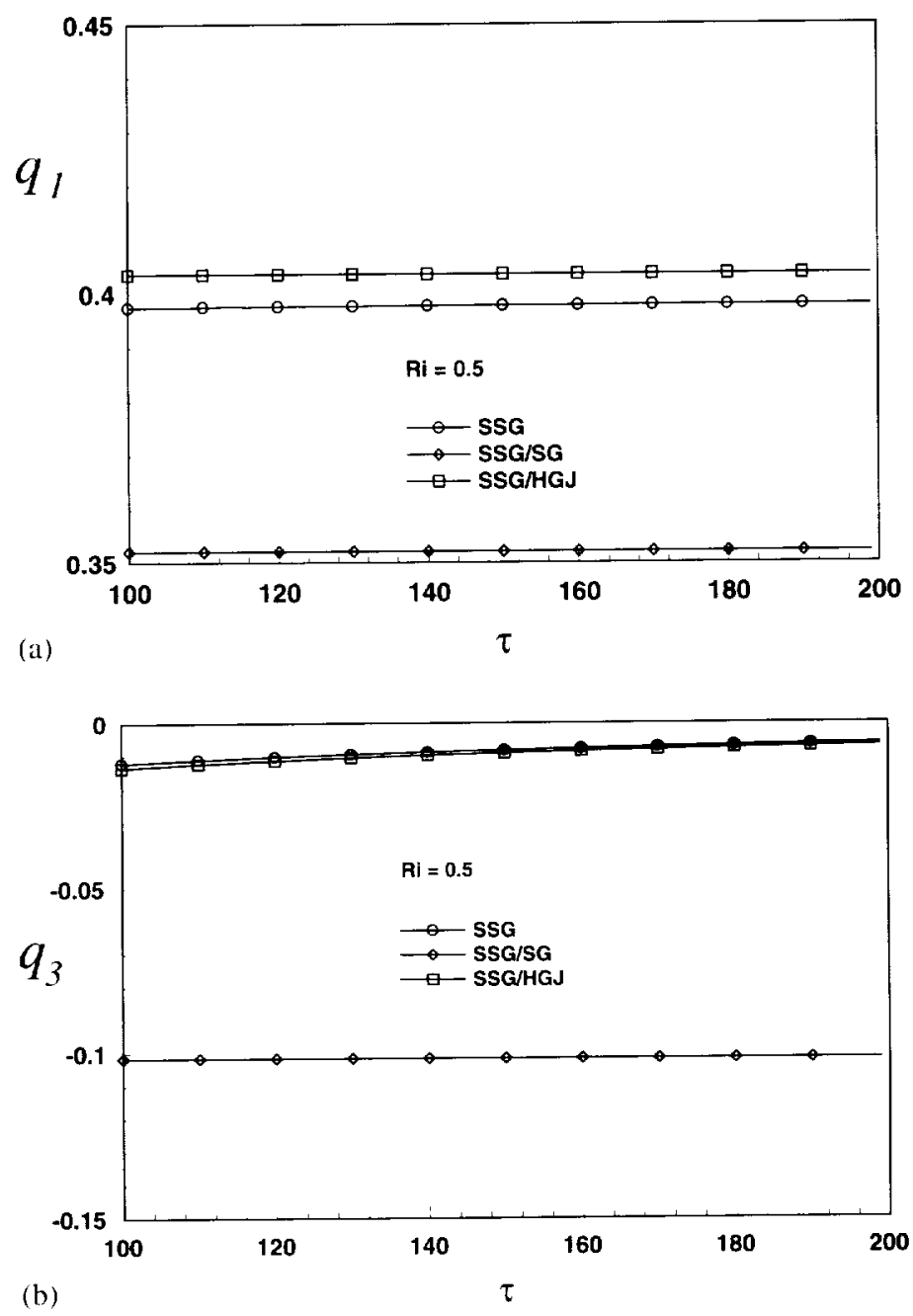

Figure 2. Variation of the components of $q_{i}$ with time assuming the JM model. 


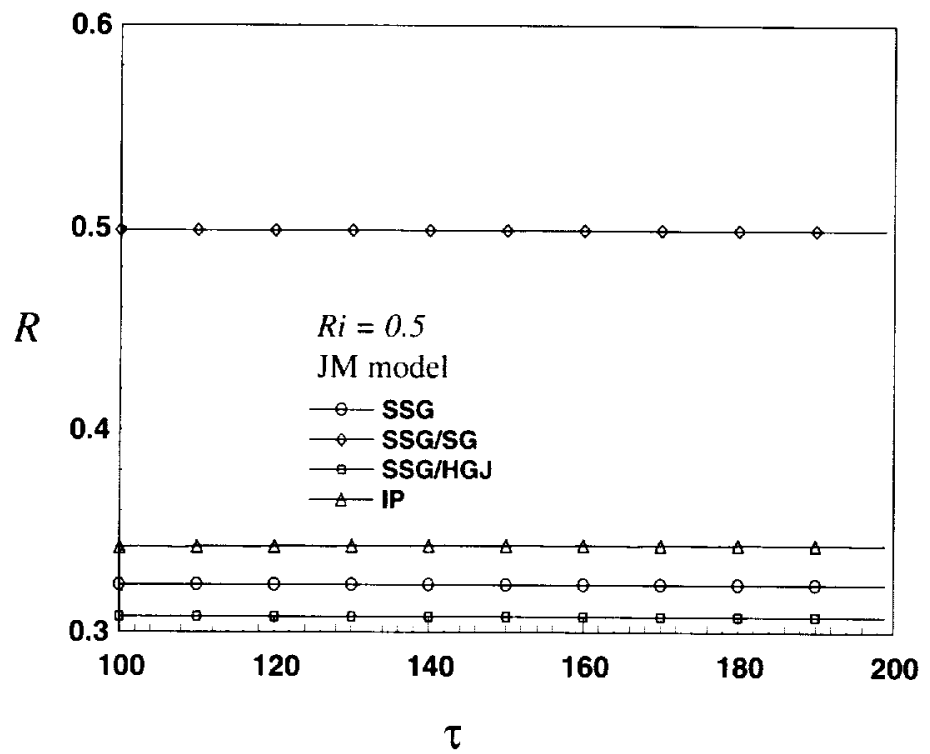

Figure 3. Variation of $R$ with lime assuming the JM model.

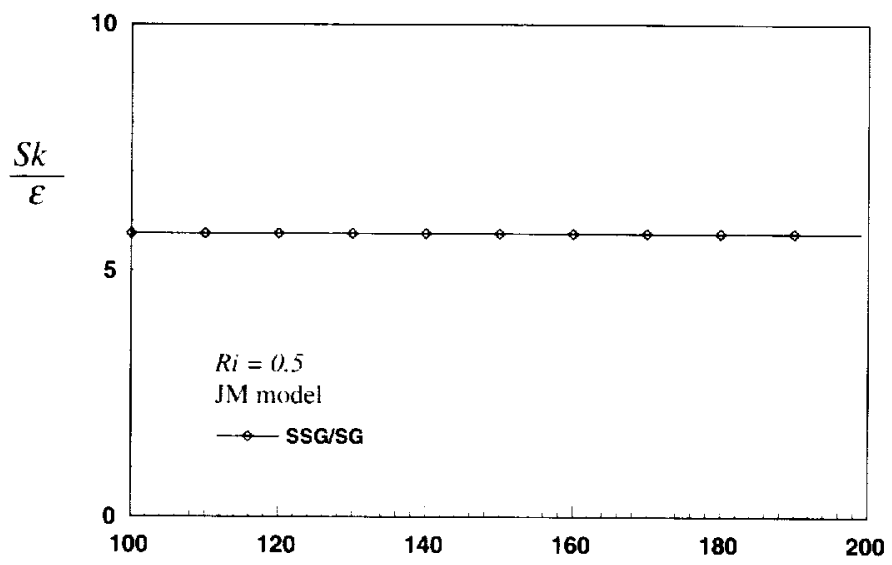

(a)

$\tau$

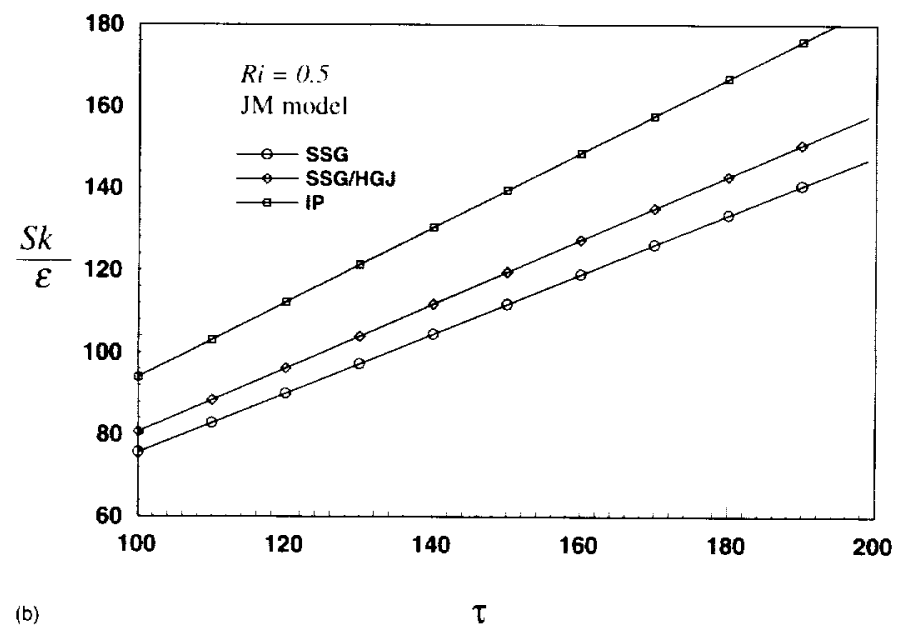

Figure 4. Variation of $S k / \varepsilon$ with time assuming the JM model. 
Therefore, plots of $S k / \varepsilon$ and $S \overline{\theta^{2}} / \varepsilon$ versus $\tau$ are also shown in Figures 4 and 5, respectively, to illustrate their behavior at large $\tau$. The results show that only the SSG/SG model yields constant $S k / \varepsilon$ (Figure $4(\mathrm{a})$ ) and $S \overline{\theta^{2}} / \varepsilon$ (Figure $5(\mathrm{a})$ ) for $\tau>100$. All other velocity models tested give an increasing $S k / \varepsilon$ and $S \overline{\theta^{2}} / \varepsilon$ as $\tau$ increases. Consequently, these results show that the combination of models SSG/SG and JM is capable of predicting equilibrium turbulence for $\tau>160$. Other velocity and thermal field models are not.

If equilibrium is taken to be given by $b_{i j} . q_{i} . R, S k / \varepsilon$ and $S \overline{\theta^{2}} / \varepsilon$ all approaching constant, then the values of $\tilde{P} / \varepsilon$ and $\tilde{P}_{H} / \varepsilon_{H}$ can be derived as follows. Substituting the trace of $(1),(10),(13)$ and (14) into (16), the following asymptotic relations are deduced for the equilibrium state parameters $\tilde{P} / \varepsilon$ and $\tilde{P}_{\theta} / \varepsilon_{\theta}$. They are

$$
\begin{aligned}
& \frac{\tilde{P}}{\varepsilon}=\frac{C_{\varepsilon 2}-1}{C_{\varepsilon 1}-1}-\frac{C_{\varepsilon 3}-1}{C_{\varepsilon 1}-1}\left(\frac{G}{\varepsilon}\right) \\
& \frac{\tilde{P}_{H}}{\varepsilon_{\theta}}=\frac{2 C_{d 3} R\left(C_{\varepsilon 2}-1\right)-2 C_{d 5} R\left(C_{\varepsilon 1}-1\right)-\left(C_{d 4}-2\right)\left(C_{\varepsilon 1}-1\right)}{\left(2-C_{d 1}-2 C_{d 2} R\right)\left(C_{\varepsilon 1}-1\right)}-\frac{2 C_{d 3} R}{2-C_{d 1}-2 C_{d 2} R}\left(\frac{C_{\varepsilon 3}-1}{C_{\varepsilon 1}-1}\right)\left(\frac{G}{\varepsilon}\right) .
\end{aligned}
$$
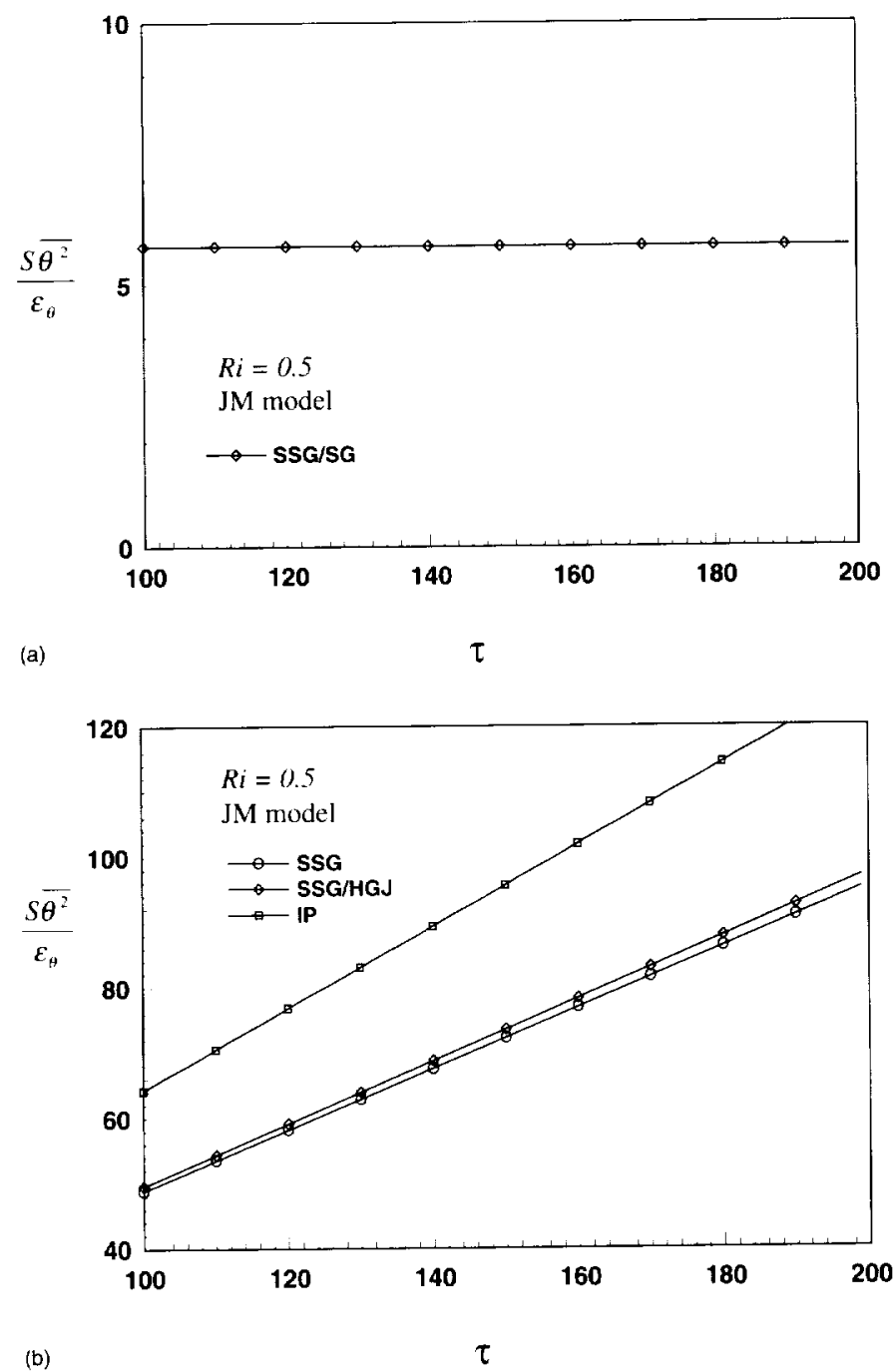

Figure 5. Variation of $S \overline{t^{2}} / \varepsilon_{i t}$ with time assuming the JM model. 
The expressions (17) and (18) relate $\tilde{P}_{H} / \varepsilon_{H}$ and $\tilde{P} / \varepsilon$ to $G / \varepsilon$ and $R$ and suggest that the equilibrium parameters $\tilde{P}_{\theta} / \varepsilon_{\theta}$ and $\tilde{P} / \varepsilon$ are constant if $G / \varepsilon$ is constant. It will be confirmed later that $G / \varepsilon$ is indeed constant at equilibrium, just as deduced by Zhao et al. (2001).

In a buoyant flow, the normal heat flux would continue to oscillate until the collapse of turbulence (Stillinger et al., 1983; Itsweire et al.. 1986). Therefore, in principle, an equilibrium state could be reached only under the condition that $G / \varepsilon$ and other relevant parameters would become constant. This calculation shows that the SSG/SG and JM combination is able to predict $\tilde{P} / \varepsilon, S k / \varepsilon, \tilde{P}_{H} / \varepsilon_{H}, R$ and $G / \varepsilon$ are constant at equilibrium. just as predicted by the analysis of Zhao et al. (2001). Unlike the case of pure shear flows, (17) and (18) also depend on the constants specified in the modeled $\varepsilon$ - and $\varepsilon_{H}$-equation. In view of this, the constant values reached at equilibrium will depend on how correctly the $\varepsilon$ - and $\varepsilon_{t+}$-equation can be modeled.

Other combinations of velocity model and $\varepsilon_{H}$-equation model will not lead to a predicted equilibrium state. An example of one such combination (SSG and NK) is shown in Figures 6-8, where the time variations of the components of $b_{i j}$ and $q_{i}$, and $R$ are plotted, respectively. It can be seen that $b_{i j}, q_{i}$ and $R$ fail to reach their asymptotic states even for very large $\tau$ (however, only the period $100<\tau<200$ ) is shown in these figures to illustrate the behavior).

\section{Effect of $\varepsilon_{\theta}$-Equation Modeling}

The above analysis on buoyant shear flows shows that at equilibrium $b_{i j}, q_{i}, \tilde{P} / \varepsilon, S k / \varepsilon, \tilde{P}_{H} / \varepsilon_{H}, R$ and $G / \varepsilon$ are constant. Therefore, the effect of the $\varepsilon_{t i}$-equation on the prediction of the equilibrium state parameters is examined next. The calculations were mostly carried out using the 2-Eq and the Reynolds-stress models. In these calculations, the heat flux model invoked was that given by (12) with $C_{3 H}$ set to zero. All $\varepsilon_{H-}$ equation models listed in Table 1 were investigated using the 2-Eq model. Only a few were examined using the Reynolds-stress models. The results for the 2-Eq model are shown in Figures 9-11, while those for the Reynolds-stress models are plotted in Figures 3-5 and 8. From this point on, only the equilibrium values of $R, S k / \varepsilon$ and $S \overline{\theta^{2}} / \varepsilon_{\theta}$ versus $R i$ are plotted. Their approach to equilibrium is not displayed because most have been shown in Figures 1-8.

The 2-Eq model calculations were carried out specifying $\operatorname{Pr}=5$. Results show that not all modeled $\varepsilon_{H}$-equation can predict equilibrium turbulence. Of the four different groups of $\varepsilon_{H}$-equations investigated, only three predict equilibrium turbulence for $0 \leq R i \leq 1$. The value of $R$ is plotted in Figure 9 for the six $\varepsilon_{\theta} \rightarrow$-equation models that yield equilibrium turbulence. Figures 10 and 11 show the equilibrium values for $S k / \varepsilon$ and $S \overline{\theta^{2}} / \varepsilon_{\theta}$, respectively, over the same range of $R i$. The $R . S k / \varepsilon$ and $S \overline{\theta^{2}} / \varepsilon_{\theta}$ thus calculated are different for different $\varepsilon_{\theta}$-equation model. In general, the values predicted by the CL model are greater than those given by the other models. Four models, GJK. JM, NK and YNT, yield very similar results, however, SSZ gives equilibrium values that are consistently higher than those obtained from GJK and JM. The correctness of these predictions will have to be verified by DNS data or experimental measurements.

Not all Reynolds-stress closures can predict equilibrium turbulence with the four groups of $\varepsilon_{\theta}$-equations listed in Table $I$ under the conditions $\mathrm{d} b_{i j} / \mathrm{d} t=0$ and $\mathrm{d} q_{i} / \mathrm{d} t=0$. Those $\varepsilon_{H}$-equations that cannot give equilibrium turbulence with the 2-Eq model also fail with the Reynolds-stress closures. Among the group of $\varepsilon_{H}$-equation models that can predict the approach to equilibrium turbulence, only the results of JM are shown becaluse equilibrium is reached after a relatively short period of time. It took much longer time for GJK and SSZ to yield equilibrium turbulence. The results shown in Figures 3-5 are for the case where $\mathrm{Pr}=5$ and $R i=0.5$ (Gerz et al. 1989). All Reynolds stress models tested clearly show that $R$ is constant (Figure 3). However, only SSG/SG give constant values for $S k / \varepsilon$ and $S \hat{\theta}^{2} / \varepsilon_{H}$ (Figures 4 (a) and $5(\mathrm{a})$ ). The parameters $S K / \varepsilon$ and $S H^{2} / \varepsilon_{\theta}$ continue to vary with time long after $R$ becomes constant for other Reynolds-stress models (Figures 4 (b) and $5(\mathrm{~b})$ ). In other words, equilibrium has not been reached. It will be shown later that when $R$. $S k / \varepsilon$ and $S \overline{\theta^{2}} / \varepsilon_{H}$ become constant, so do $\tilde{P} / \varepsilon, \tilde{P}_{\theta} / \varepsilon_{\theta}$ and $G / \varepsilon$. Other $\varepsilon_{H}$-equation models fail to give equilibrium prediction. A representative plot of this behavior is shown in Figure 8.

According to the heat transfer studies made by the researchers of the nine different modeled $\varepsilon_{t \theta}$ equaltions, setting $C_{d 2}=0$ gives better results for wall-bounded flows, while setting $C_{d l}=0$ yields good agreement with the turbulence statistics of free shear flows. The other constants are found to have litthe effect on the calculations as long as they do not differ by much. Therefore, it appears that there is no one single set of constants that could give reasonably good results for both wall-bounded and free 


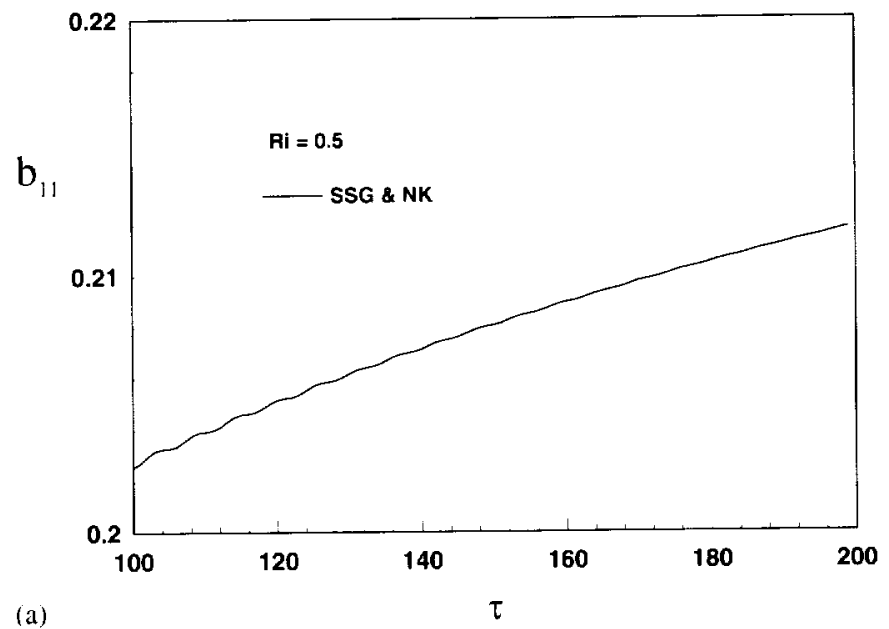

(a)
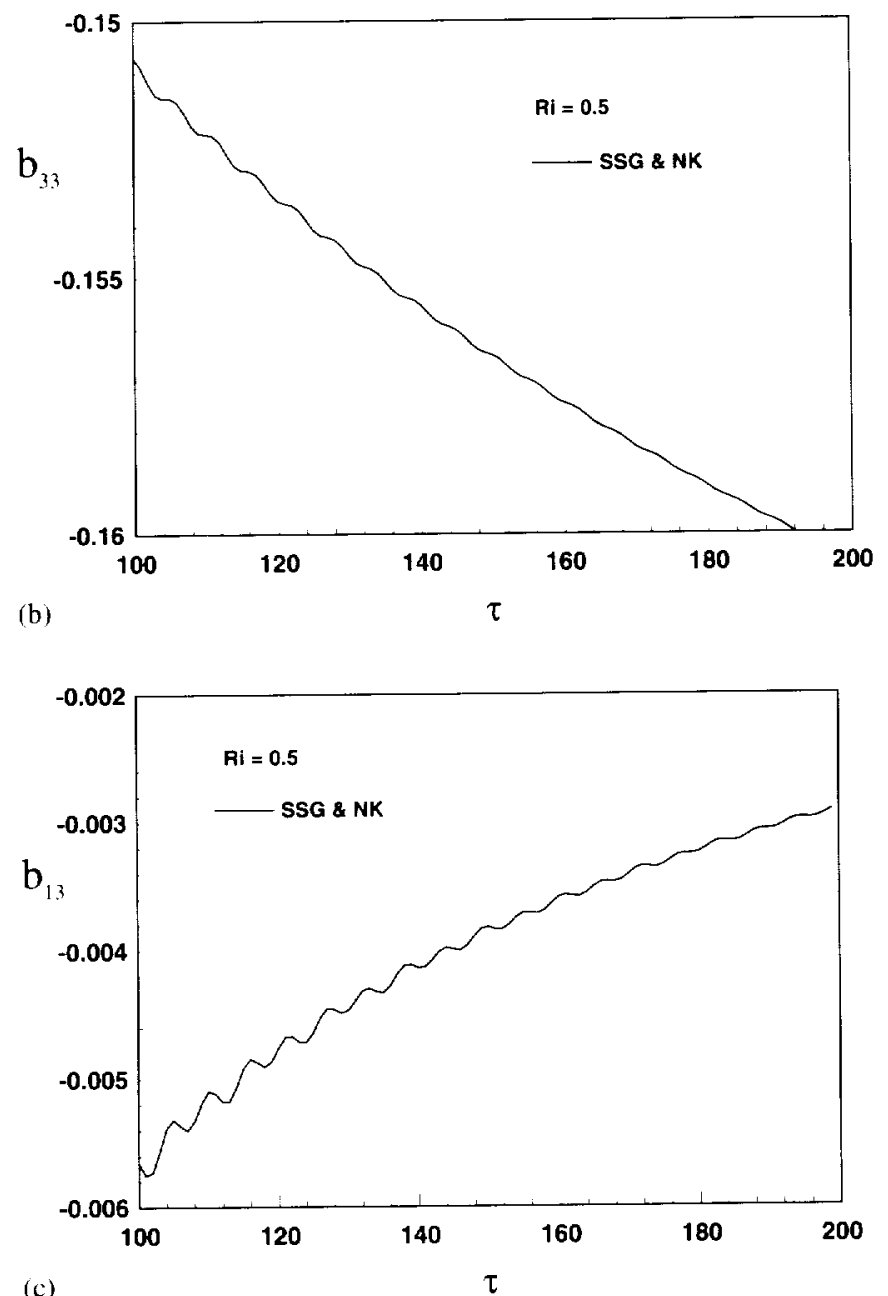

Figure 6. Variation of the components of $b_{i j}$ with time for the SSG and NK models.

shear flows. This is especially true when buoyancy-induced gravity waves (Sommer et al., 1997) and buoyant jets and plumes (Craft, 1991) are considered. In fact. Craft (1991) found that the constants $C_{d 2}, C_{d 4}$ and $C_{d 5}$ should be modified to make them dependent on $R$ and the stress invariants. With 

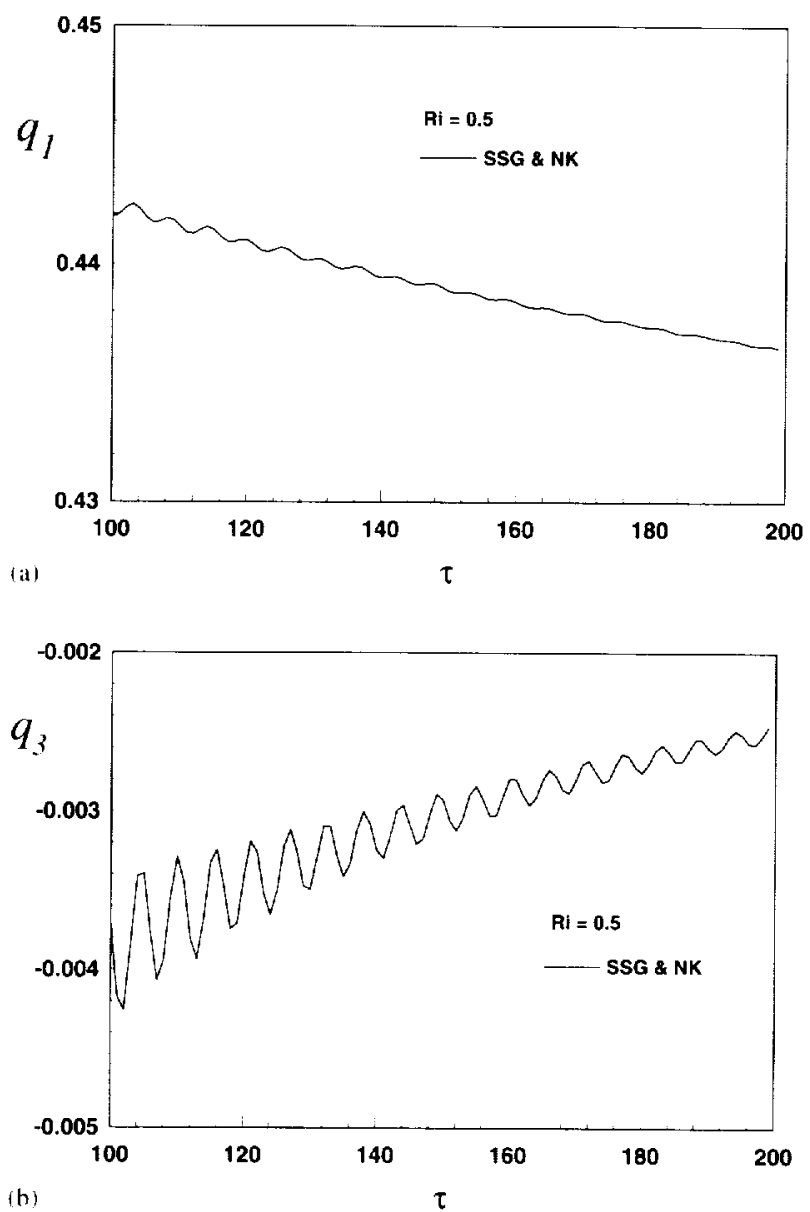

Figure 7. Variation of the components of $q$ with time for the SSG and NK models.

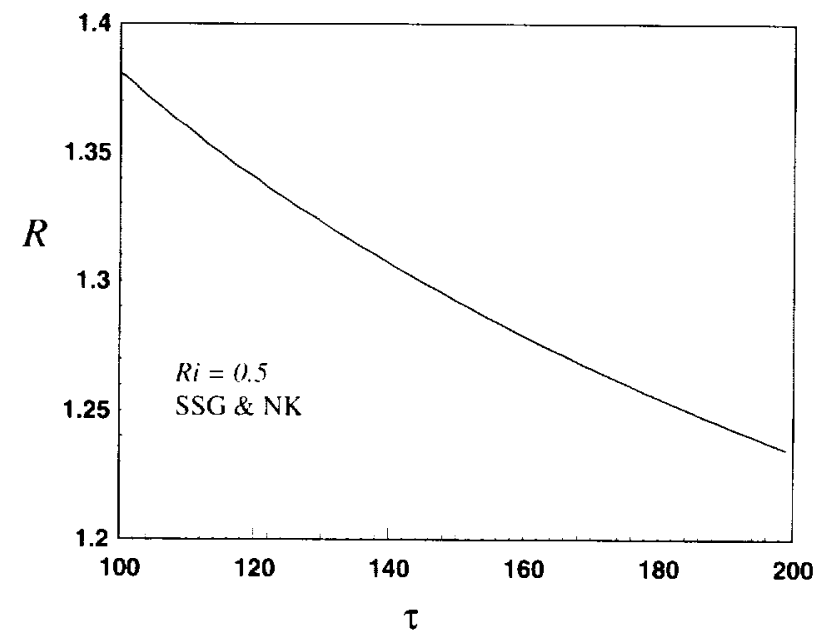

Figure 8. Variation of $R$ with time for the SSG and NK models.

this understanding, the inability of NLL, EL, AY, NK and YNT to predict equilibrium turbulence compared with JM is not surprising. As for $\mathrm{CL}$, it assumes $C_{d 3}=0$. Therefore, it is reasonable to expect CL to perform differently from JM. The only difference between GJK, SSZ and JM is in the values assigned to the constants $C_{d 2}$ to $C_{d 5}$. For example. GJK assumes a value of 1.80 for $C_{d 5}$, while JM 


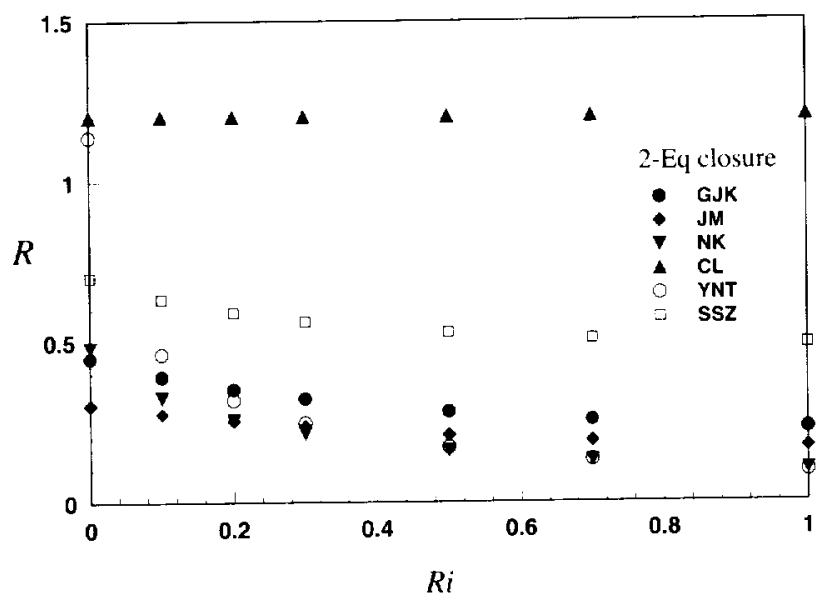

Figure 9. Variation of $R$ with $R i$ at equilibrium for different $\varepsilon_{4}$-equation models.

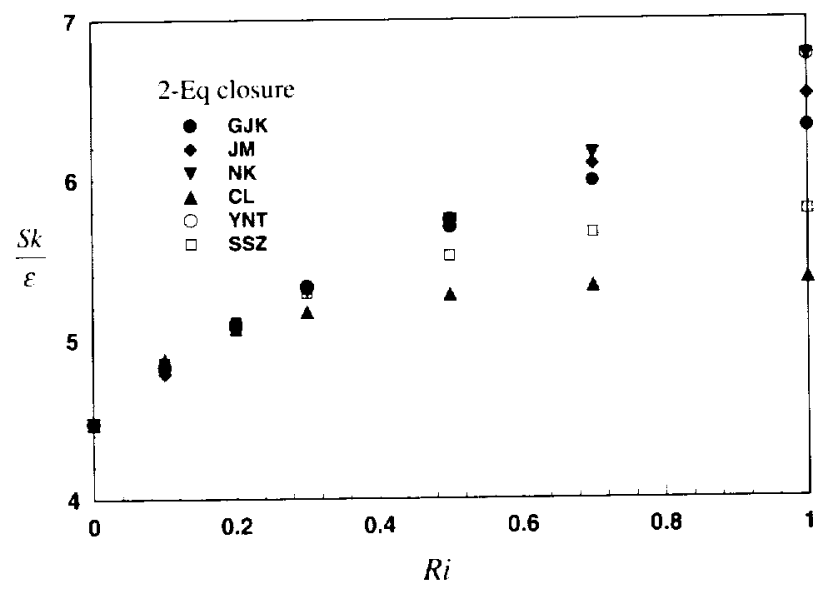

Figure 10. Variation of $S k / \varepsilon$ with $R i$ at equilibrium for different $\varepsilon_{t}$-equation models.

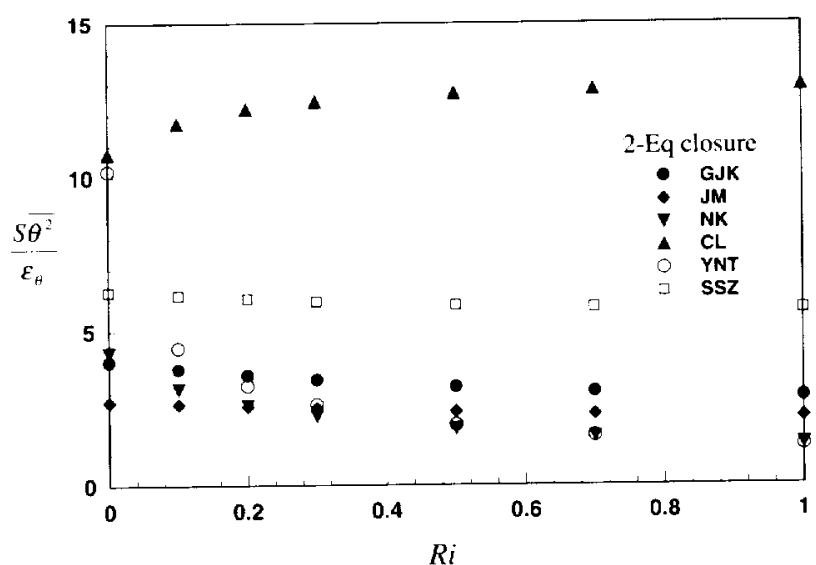

Figure 11. Variation of $S \overline{H^{2}} / \varepsilon_{*}$ with $R i$ at equilibrium for different $\varepsilon_{n}$-equation models.

takes on 0.52 . On the other hand, SSZ and JM invoke different constants for $C_{d 2}$ and $C_{d 5}$. Since all three models, GJK, JM and SSZ, are formulated for free turbulence, the present study shows that the choice of constants is important. Furthermore, the constants play a crucial role in the prediction of 


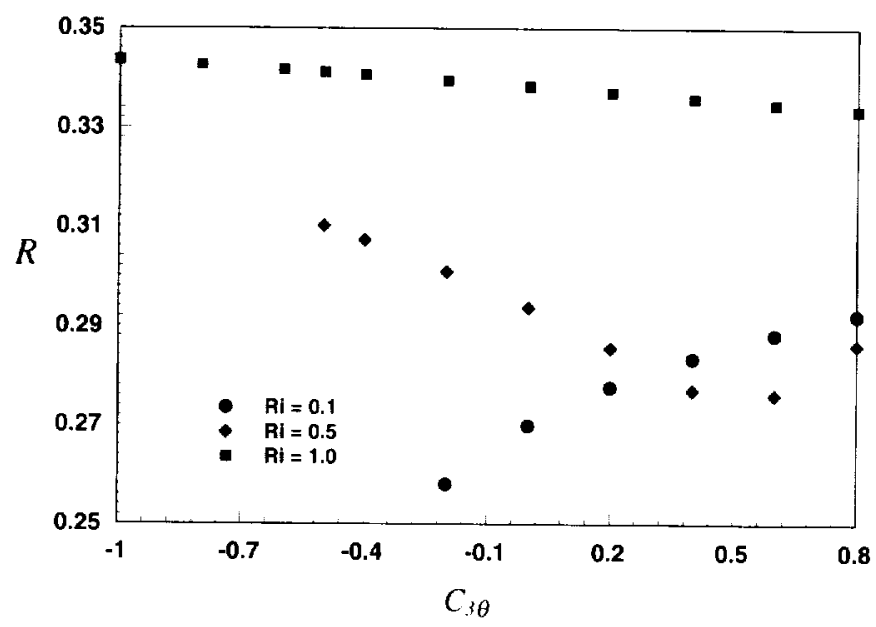

Figure 12. Variation of $R$ with $C_{3}$ at equilibrium for different $R i$.

the approach to equilibrium turbulence and of the asymptotic values of the state parameters in buoyant shear flows.

Unlike the modeled $\varepsilon$-equation in the pure shear flow case, the modeled $\varepsilon_{\theta}$-equation affects the predicted values of the state parameters and directly influences the prediction of the approach to equilibrit $\mathrm{m}$ turbulence.

\section{Effect of Heat Flux Modeling}

Having examined the effect of the $\varepsilon_{\theta}$-equation model, the next task is to investigate the effect of $\Phi_{\theta i}$ modsling. The above analysis is carried out with $C_{3 \theta}=0$ in $(12)$. In this section the effect of including this term in the $\Phi_{t i}$ model on the prediction of equilibrium turbulence is studied. It should be pointed out that, in the calculations presented above, where $C_{3 H}=0$ was specified in (12), both the IP and SSG closures using JM yield equilibrium turbulence where $b_{i j}, q_{i}, R, S k / \varepsilon$ and $S \overline{\theta^{2}} / \varepsilon_{t}$ are constant. Equilibrium turbulen ze was predicted for the range of $R i$ investigated. Therefore, only these two closures are investigated in detail in this section. The modeled equations solved are the same as before, except that the $C_{3 H}$ term is retained in (12). The case studied is the DNS turbulent buoyant shear flow of Gerz et al. (1989) where $\operatorname{Pr}=5.0$ and $R i=0.5$. When the $C_{3 \theta}$ term is added to (12), both IP and SSG closures predict equilibrium turbulen:e provided the value of $C_{2 \theta}$ was modified to be 0.8 . As for the value of $C_{3 H}$. it was found that a range of values would lead to the prediction of equilibrium turbulence. The plot of $R$ versus $C_{3 \theta}$ for the SSG cl ssure using JM is shown in Figure 12. The figure only shows the values of $R$ for $R i=0.1,0.5$ and 1.0. This range of $C_{3 H}$ values is not consistent with those recommended by Launder (1978). Therefore, these calc 1 lations tend to show that, when the pressure-scrambling term is modeled with the $C_{3 H}$ term included, the IP or the SSG closure still predicts an approach to equilibrium turbulence for all values of $R i$ tested for a given set of $C_{2 H}$ and $C_{3 H}$. However, the value of $R$ calculated differs depending on the choice of collstants. In other words, the addition of the $C_{3+}$ term to (12) has an appreciable effect on the prediction of equilibrium values. This effect is not desirable because it varies with the choice of $C_{3 \theta}$. Furthermore, it is not known which $C_{3 \theta}$ would be more suitable for the developing stage of buoyant shear flows and for wall shear flows.

\section{Effect of Pressure-Strain Models}

The velocity closures are examined next and it is mainly concentrated on the effect of the pressure-strain models. Altogether two different pressure-strain models have been examined. These are the linear IP and the nonlinear SSG model. Calculations were carried out using the JM model for the $\varepsilon_{\theta}$-equation. The calculated equilibrium state parameters for different $R i$ are shown in Figures 13-16. All models (2-Eq. I?, 
Turbulence Modeling Effects on the Prediction of Equilibrium States of Buoyant Shear Flows

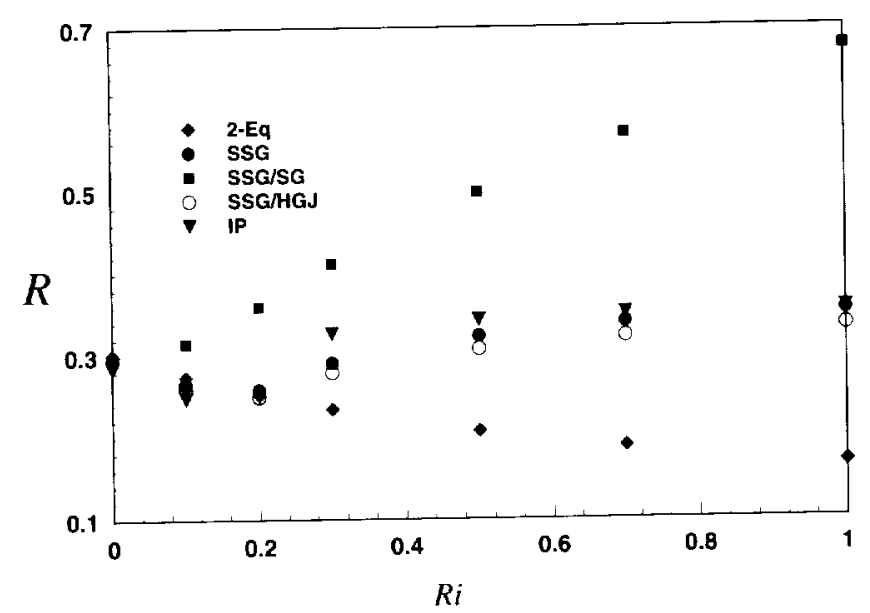

Figure 13. Variation of $R$ with $R i$ at equilibrium for different velocity models.

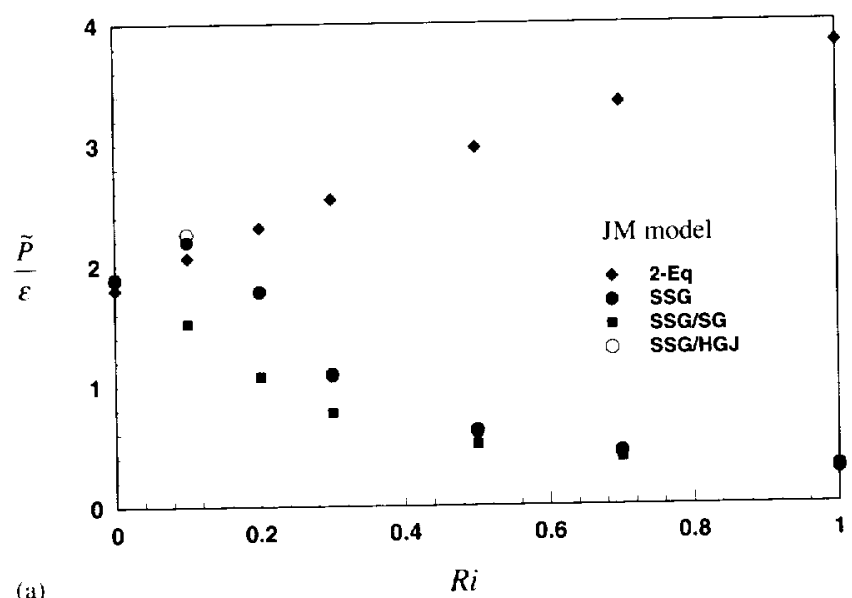

(a)

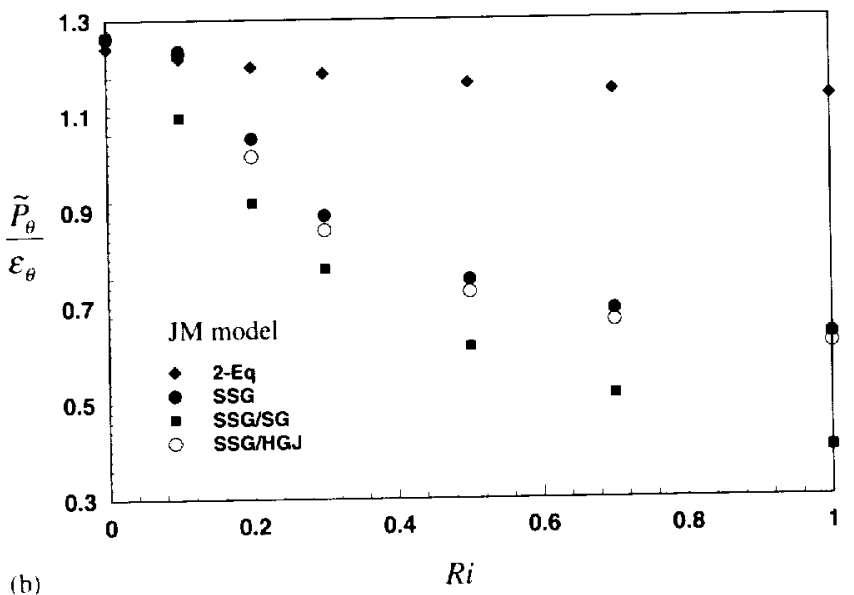

Figure 14. Variation of (a) $\tilde{P} / \varepsilon$ and (b) $\tilde{P}_{i t} / \varepsilon_{i j}$ with $R i$ at equilibrium for different $\varepsilon_{i j}$ models.

SSG, SSG/HGJ and SSG/SG) tested can predict constant $b_{i j}, q_{i}$ and $R$. However, only the 2-Eq and SSG/SG closure are capable of predicting equilibrium behavior with $S k / \varepsilon$ and $S \theta^{2} / \varepsilon_{\theta}$ also becoming constant (Figure 16). Together, these results show that IP, SSG and SSG/HGJ fail to predict the approach to equilibrium turbulence. In the case of IP and SSG, anisotropic modeling of the dissipation rate tensor is not 


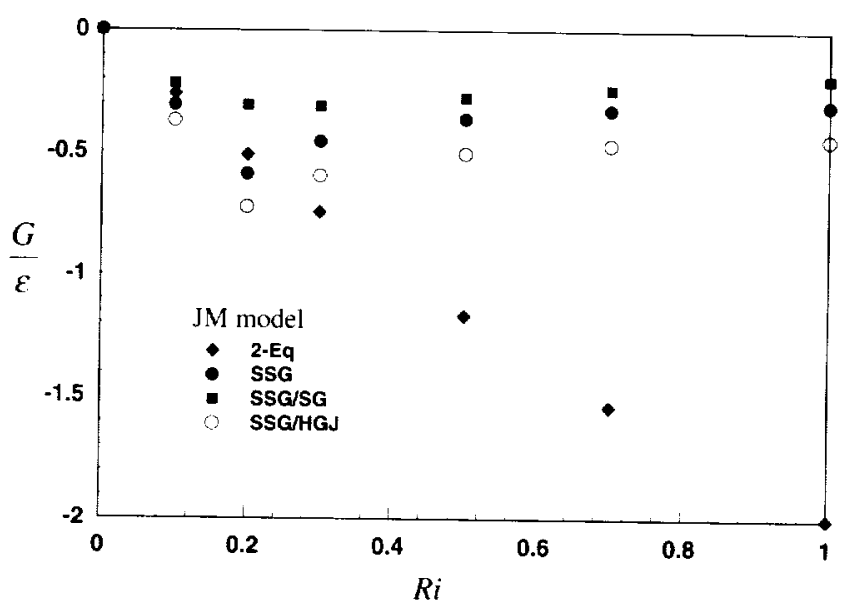

Figure 15. Variation of $G / \varepsilon$ with $R i$ at equilibrium for different dissipation rate models.

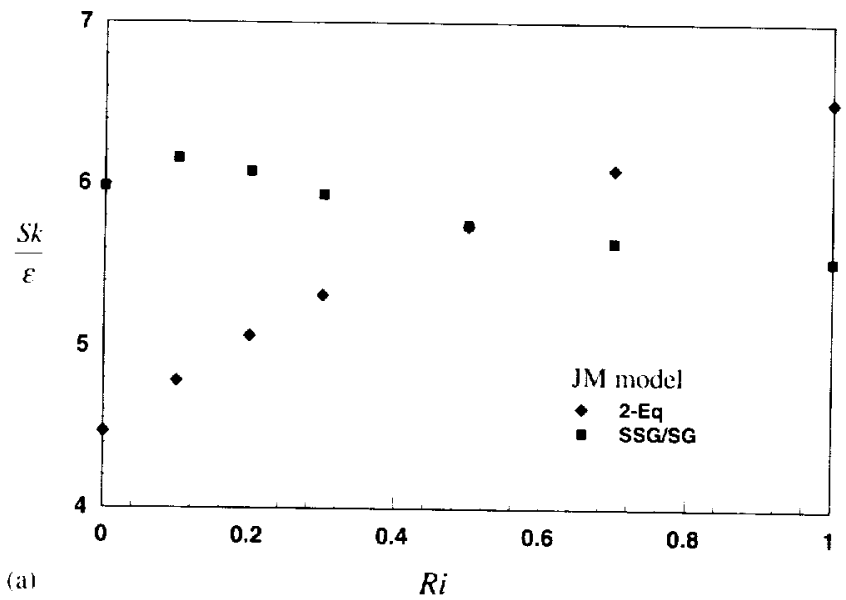

(a)

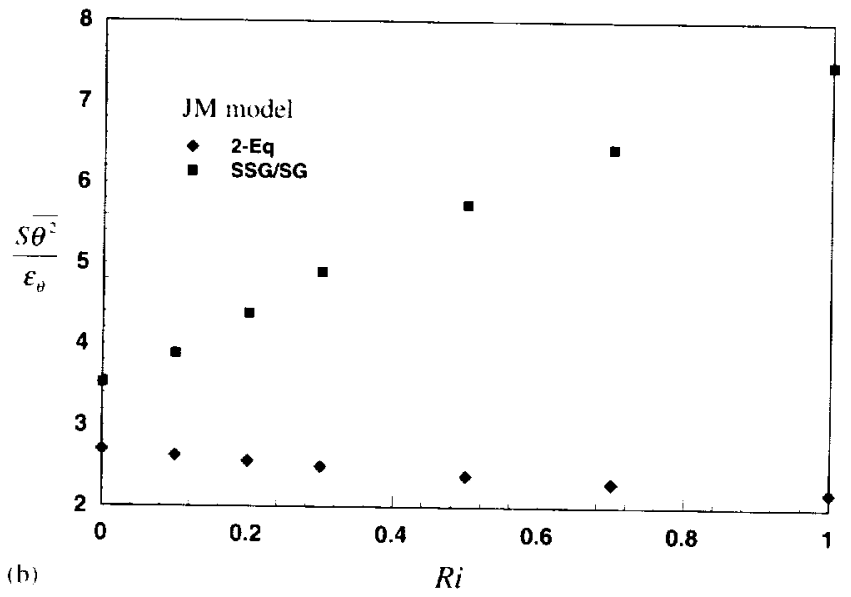

Figure 16. Variation of (a) $S k / \varepsilon$ and (b) $S \overline{A^{2}} / \varepsilon_{i}$ with $R i$ at equilibrium for two different velocity models.

invoked. On the other hand, anisotropic modeling of the dissipation rate tensor is assumed in SSG/HGJ and $\mathrm{SSG} / \mathrm{SG}$. The differences are in the models assumed in $d_{i j}$ and in the modeled $\varepsilon$-equation. In SSG/HGJ the anisotropy is expressed as a function of $b_{i j}$, while SSG/SG represents $d_{i j}$ in terms of $S_{i j}$ and $W_{i j}$ and solved a different $\varepsilon$-equation. The present results seem to imply that either the form used in SSG/SG to account for 
dissipation anisotropy or the $\varepsilon$-equation assumed is more appropriate. It will be shown in the next section that a simple modification of the $\varepsilon$-equation is sufficient to render IP and SSG valid in the prediction of the approach to equilibrium turbulence. This may not be surprising since the equilibrium analysis did not need to account for dissipation rate anisotropy.

Asymptotic values of $\tilde{P} / \varepsilon$ and $\tilde{P}_{H} / \varepsilon_{\theta}$ thus determined for different $R i$ are shown in Figure 14 . In the case of $\tilde{P} / \varepsilon$, the 2 -Eq prediction is of opposite trend to that given by the SSG-type closures. Instead of a decreasing trend for increasing $R i$, the 2 -Eq result shows an increasing behavior with $R i$. According to the $\mathrm{SSG} / \mathrm{SG}$ calculations, the heat flux contribution is quite small and is positive, while the shear component is large and is negative. Therefore, the trend displays by the 2-Eq closure could not be correct. As for the behavior of $\tilde{P}_{\theta} / \varepsilon_{\theta}$ (Figure $14($ b) ), again the SSG-type closures yield a decreasing trend similar to that of $\tilde{P} / \varepsilon$. However. the value reached at $R i=1$ is much smaller than that given by the 2 -Eq closure. This implies that stratification suppresses the production of $\overline{\theta^{2}}$ more strongly than its dissipation. On the other hand, the 2-Eq closure predicts that the production of $\overline{\theta^{2}}$ and its dissipation are roughly equal for $R i>0.5$. The behavior of $G / \varepsilon$ with $R i$ is shown in Figure 15. Just as before. the values predicted by the SSG-type closures are quite similar, while those given by the 2-Eq closure essentially decrease linearly with $R i$.

\section{Effect of Anisotropic Dissipation Rate Modeling}

The next task is to examine the effect of anisotropic dissipation modeling on the calculation of equilibrium states. Three different dissipation models are considered. The first is the isotropic model (5). the second is that given by (6) and (7), while the third is provided by (6), (8) and (9). The IP and SSG closures are used to carry out the calculation. In all these calculations, the JM model is invoked for the $\varepsilon_{\theta}$-equation and (12) is solved with $C_{34}=0$. Again, the case calculated has the same initial conditions as those given by Gerz et al. (1989).

From the above analysis, it is known that different dissipation rate models give rise to different predictions of the equilibrium values. The reason why the SSG/SG closure can predict equilibrium turbulence while the IP, SSG and SSG/HGJ closures cannot is, perhaps, due to a difference in the modeled $\varepsilon$-equation invoked by the closure models. The SSG/SG closure assumes (11) while IP, SSG and SSG/HGJ invoke (10). The difference between these two equations is the additional $d_{i j} S_{i j}$ term in (11). In conjunction with (8) and (9), the leading term of $d_{i j} S_{i j}$ is given by a constant times $k S_{i j} S_{i j}$. This term is similar to a vortex stretching term introduced by Bernard and Speziale (1992) to drive the flow to a production-balance-dissipation equilibrium with bounded energy states. Thus (11) could be interpreted as a first attempt to account for anisotropic dissipation, yet also serves to strain the vorticity by mean shear. The question then is could such a term in the modeled $\varepsilon$-equation be sufficient to remedy the shortcoming of the IP and SSG closures without having to invoke an anisotropic dissipation rate model in (1) such as given by (8) and (9)? An attempt is made to answer this question here.

An $\varepsilon$-equation with an additional term to account for vortex stretching has been proposed by Bernard and Speziale (1992). It is derived from the equation governing the behavior of enstrophy $\omega^{2}=\omega_{i} \omega_{i}$ in isotropic turbulence and the identity $\varepsilon=v \omega^{2}$. Thus derived, the additional term depends on the fluid kinematic viscosity, $v$, and the modified $\varepsilon$-equation for turbulent buoyant shear flows becomes

$$
\frac{\mathrm{d} \varepsilon}{\mathrm{d} t}=C_{\varepsilon 1} \frac{\varepsilon}{k} \tilde{P}+\frac{7}{3 \sqrt{15}} \frac{C_{\varepsilon}^{*}}{\sqrt{\nu}} \varepsilon^{3 / 2}-C_{\varepsilon 2} \frac{\varepsilon^{2}}{k}-C_{\varepsilon \cdot 3} \frac{\varepsilon}{k} \beta g_{i} \overline{u u_{i} \theta} .
$$

The model constants take on the following values: $C_{\varepsilon 1}=1.44, C_{\varepsilon 2}=1.83, C_{\varepsilon, 3}^{*}=0.01$ and $C_{\varepsilon 3}=1.44$. The vortex-stretching term renders the solution a function of the initial turbulent Reynolds number Re (So et al., 1999). This is obviously not the case, even for pure shear flows. An alternative to this proposal is given by (11). The additional term in (11) could be simplified to give a term quite similar to the one proposed by Bernard and Speziale (1992) and yet not dependent on the initial turbulent $R e$. Since the leading term in $\varepsilon d_{i j} S_{i j}$ is a constant times $k S_{i j} S_{i j}$ and $k^{2} S_{i j} S_{i j} / \varepsilon^{2}$ is a constant for homogeneous shear flows, the term $k^{2}\left(S_{i j} S_{i j}\right)^{2}$ could be related to $\varepsilon^{2} S_{i j} S_{i j}$. Thus modified, the $\varepsilon$-equation (11) could be written as

$$
\frac{\mathrm{d} \varepsilon}{\mathrm{d} t}=C_{k 1} \frac{\varepsilon}{k} \tilde{P}+C_{+4} \sqrt{2}\left(S_{i j} S_{i j}\right)^{1 / 2} \varepsilon-C_{\varepsilon 2} \frac{\varepsilon^{2}}{k}-C_{\varepsilon 3} \frac{\varepsilon}{k} \beta g_{i} \overline{u_{i} \theta} .
$$




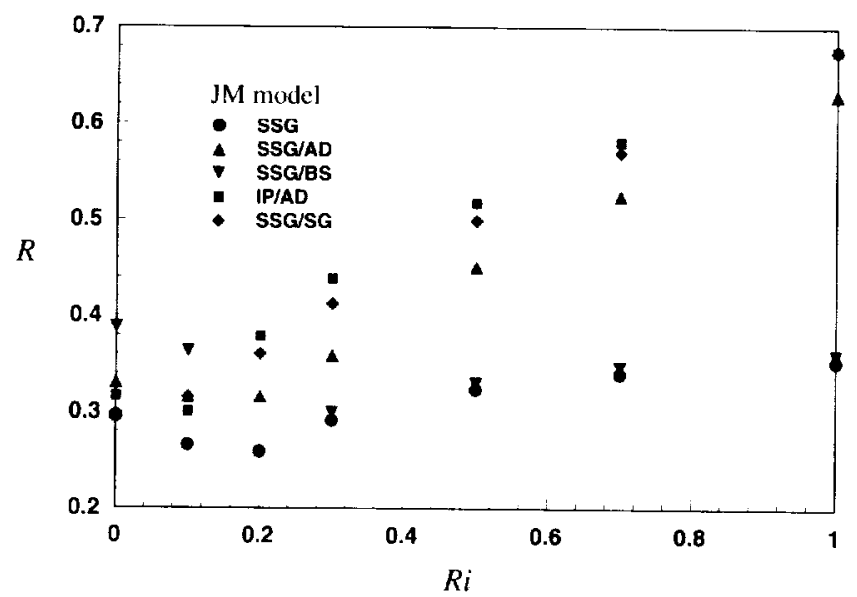

Figure 17. Variation of $R$ with $R i$ at equilibrium for different $\varepsilon$-equations
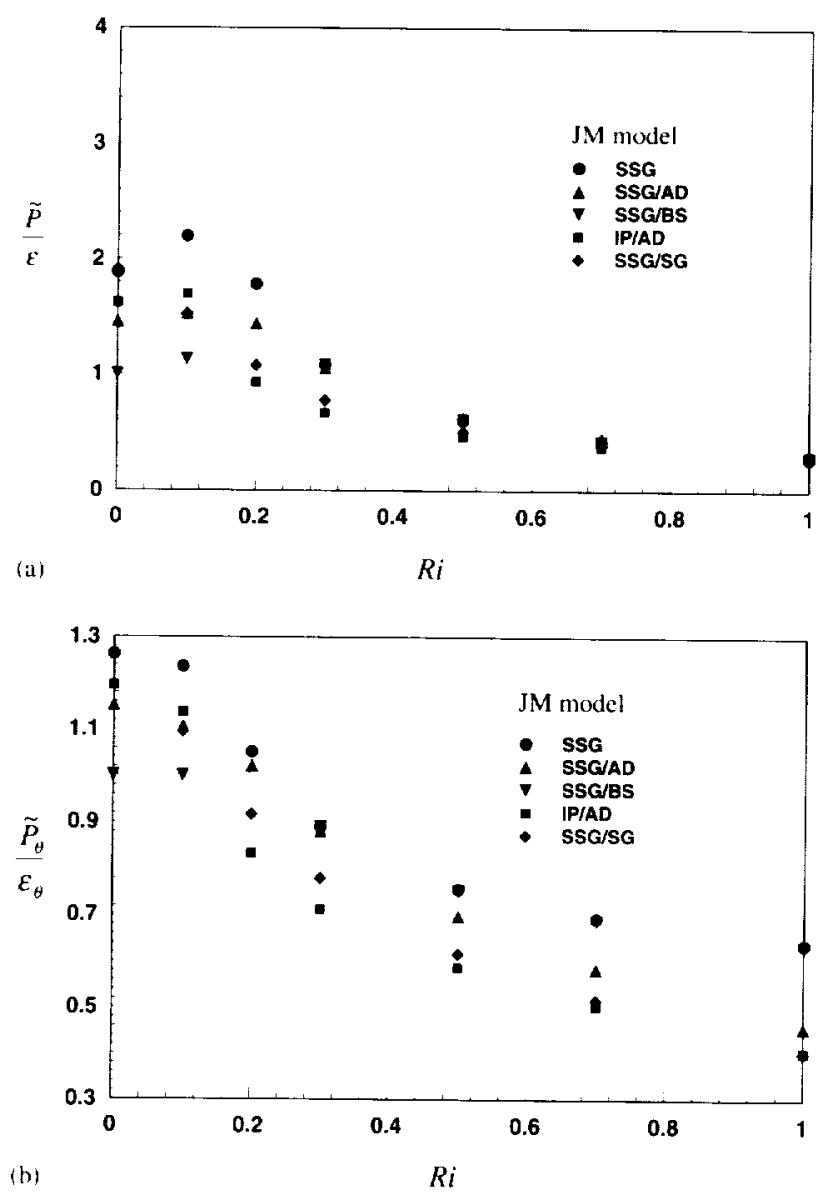

Figure 18. Variation of (a) $\tilde{P} / \varepsilon$ and (b) $\tilde{P}_{H / \xi_{H}}$ with $R i$ at equilibrium for different $\varepsilon$-equations.

where $C_{\varepsilon 4}=0.042$ is a model constant that yields the best predictions for pure shear flows.

In the following, AD is used to denote the $\varepsilon$-equation (20) and the SSG closure that solves (20) is designated as SSG/AD. In this closure the dissipation rate tensor is still modeled by (5). Thus, SSG/AD represents a first attempt to approximate the effect of anisotropic dissipation. If the results come out to be similar to those given by SSG/SG, then there is no need to solve (6), (8) and (9). The performance of (20) 
Turbulence Modeling Effects on the Prediction of Equilibrium States of Buoyant Shear Flows

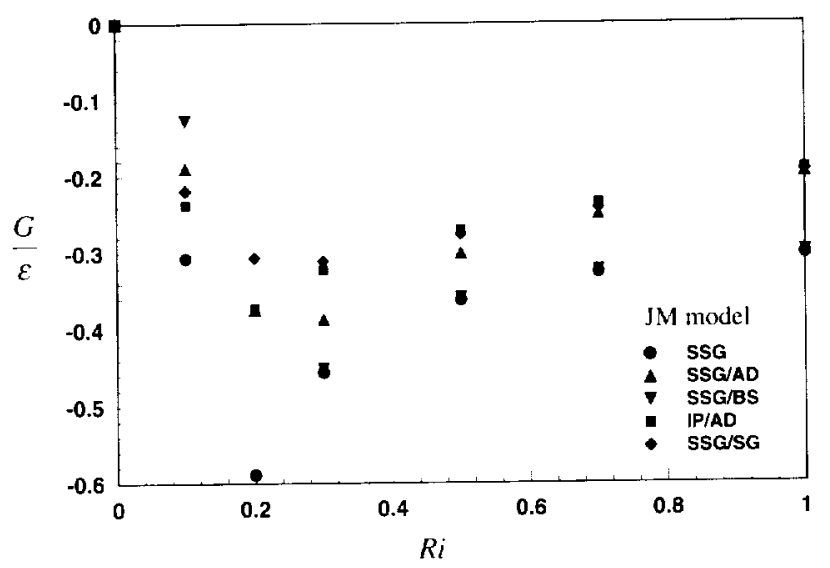

Figure 19. Variation of $G / \varepsilon$ with $R i$ at equilibrium for different $\varepsilon$-equations.
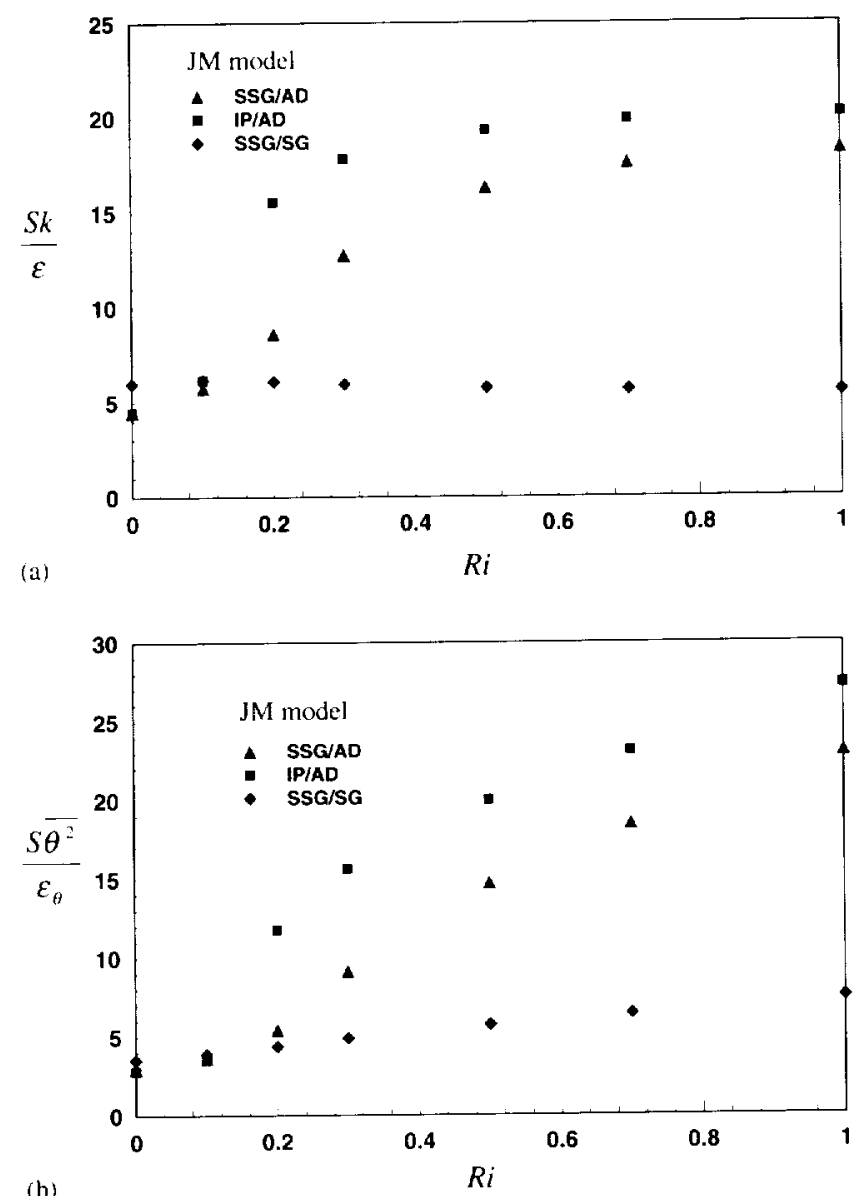

Figure 20. Variation of (a) $S k / \varepsilon$ and (b) $S A^{2} / \varepsilon$, with $R i$ at equilibrium for different $\varepsilon$-equations.

has been validated against the measured decay of $k / k_{0}$ with time (Tavoularis and Karnik, 1989) by So $e t a l$. (1999), where $k_{0}$ is the initial value of $k$. Other closures used to calculate the same flow include 2-Eq, IP, SSG, SSG/HGJ and SSG/BS. The SSG/BS closure is the same as SSG but solves the $\varepsilon$-equation (19) instead of (10). The SSG/AD and SSG/BS closures were found to yield good agreement with experimental measurements. All other closure models did not. However, the SSG/BS closure suffers from the shortcoming of being dependent on the initial $R e$. This cannot be the case for long time. 
With a new proposal to account for anisotropic dissipation. the ability of the SSG model to predict equilibrium states of turbulent buoyant shear flows is greatly improved. If (20) instead of (10) is used with the IP and SSG models, the calculations show that they can predict the approach to equilibrium turbulence much like the SSG/SG closure, i.e. at equilibrium $\tilde{P} / \varepsilon, \tilde{P}_{H} / \varepsilon_{t+}, R, G / \varepsilon, S k / \varepsilon$ and $S \theta^{2} / \varepsilon_{t i}$ all approach constant. The results of these calculations are shown in Figures 17-20. Altogether, five closure calculations are shown. they are SSG. SSG/AD, SSG/SG, IP/AD and SSG/BS. The variations of $R, \tilde{P} / \varepsilon, \tilde{P}_{t /} / \varepsilon_{t,}, G / \varepsilon, S k / \varepsilon$ and $S \overrightarrow{H^{2}} / \varepsilon_{H}$ with $R i$ are plotted in Figures 17, 18(a), (b). 19. and 20(a). (b). respectively. From these results, it is obvious that SSG/BS cannot predict equilibrium turbulence with $R, S k / \varepsilon$ and $S \theta^{2} / \varepsilon_{1 /}$ becoming constant simultaneously, even though $\tilde{P} / \varepsilon, \tilde{P}_{H} / \varepsilon_{H}$ and $G / \varepsilon$ also achieve constant behavior. It appears that the $R$ prediction of SSG/AD is very close to that of SSG/SG. This is also true for IP/AD. On the other hand, the SSG/AD predictions of $S k / \varepsilon$ and $S \overline{\theta^{2}} / \varepsilon_{\theta}$ are higher than those given by SSG/SG by a factor of 2 . The predicted behavior and magnitudes of $\tilde{P} / \varepsilon, \tilde{P}_{H} / \varepsilon_{t}$ and $G / \varepsilon$ given by SSG $/ \mathrm{SG}, \mathrm{SSG} / \mathrm{AD}$ and IP/AD are very similar. It can be seen that all closures tested give a decreasing trend for $\tilde{P} / \varepsilon$ and $\tilde{P}_{t i} / \varepsilon_{i \prime}$ with $R i$, but an increasing trend with $R i$ for $R$. The predictions, therefore. imply that the production of $k$ and $\overline{\theta^{2}}$ decreases faster than their dissipation as $R i$ increases. These results tend to suggest that the modeling of $\varepsilon_{i j}$ has an effect on the prediction of equilibrium turbulence. It is important to account for anisotropic dissipation, however. it is not necessary to model the effect in $\varepsilon_{i j}$ directly; it could be approximately accounted for in the $\varepsilon$-equation.

\section{Conclusions}

This investigation of the effects of turbulence models on the prediction of equilibrium states of buoyant shear flow leads to the following conclusions:

(a) The equilibrium turbulence state defined by $\mathrm{d} b_{i j} / \mathrm{d} t=0$ and $\mathrm{d} q_{i} / \mathrm{d} t=0$ leads to $R, \tilde{P} / \varepsilon, \tilde{P}_{H} / \varepsilon_{H} . G / \varepsilon$ and $S k / \varepsilon$ becoming constant at equilibrium, consistent with the analysis of Zhao $e t$ al. (2001).

(b) Among the different modeled $\varepsilon_{t}$-equations considered, only three groups could lead to the prediction of equilibrium turbulence when a 2 -Eq closure is used. If the velocity field is closed by SSG/SG, only one group of models could give rise to cquilibrium turbulence. In this group only the $\mathbf{J M}$ model would predict equilibrium turbulence after at relatively short period of time.

(c) All other closures, IP, SSG, SSG/SG and SSG/HGJ, even in conjunction with JM. could not predict the approach to equilibrium turbulence.

(d) It is found that variations in the modeling of the pressure-strain tensor and the pressure-scrambling vector have little or no effect on the prediction of the approach to equilibrium turbulence. The actual predictions of the equilibrium values may differ slightly though.

(e) The SSG/AD and IP/AD closures can predict the approach to equilibrium turbulence just like that of SSG/SG. This suggests that a simple accounting for anisotropic dissipation behavior could be accomplished through a suitable modification of the $\varepsilon$-equation.

\section{References}

Abid. R.. and Speziale. C.G. (1993). Predicting equilibrium stales with Reynolds stress closures in channel fow and homogeneous shear flow. Phys. Fluids A, 5, 1776-1782.

Bernard. P.S.. and Speziale. C.G. (1992). Bounded encrgy states in homogeneous turbulent shear flow - in alternative view. J. Fheids Eng. 114. 29-39.

Craft. T.J. (1991). Second-moment modeling of turbulent scalar transport. Ph. D. thesis. Mechanical Engineering Department. UMIST, Manchester.

Craft. T.J.. and Launder, B.E. (1989). A new model for the pressure/scalar-gradient correlation and its application to homogeneous and inhomogeneous free shear flows. Seventh Symposium of Turbulent Shear Flows, Paper No. 124. Stanford. CA.

Elghobashi. S.E., and Latunder. B.E. (1983). Turbulent time scales and the dissipation ratio of temperature variance in the thermal mixing layer. Phys. Fluids 26, 2415-2419.

Ger/. T.. and Schumann. U. (199)1, Direct simulation of homogeneous turbulence and gravily waves in sheared and unsheared stratilied flows. In Turbulence Shear Flows 7 (F. Durst et il.. ed.) pp. 27-46. Springer Verlag. Berlin. 
Gerz. T.. Schumann. U., and Elghobashi. S.E. (1989). Direct numerical simulation of stratified homogeneous turbulent shear flow. J. Fluid Mechl, 200, 563-594.

Gibson. M.M., Jones, W.P., and Kanellopoulos, V.E. (1987). Turbulent temperature mixing layer: measurement and modeling. Proceedings of the 6th Srmposiam on Turbalem Shear Flons, Toulouse.

Hallback, M., Groth, J., and Johansson. A.V. 1990). An algebraic model for nonisotropic turbulent dissipation rate in Reynolds-stress closures. Phws. Fluids A 2, $1859-1866$.

Harris, V.G. Graham, J.A., and Corsin, S. (1977). Further experiments in nearly homogeneous turbulent shear flow. $J$. Fluid $M e d h$. $\mathbf{8 1}, 657-687$.

Itsweire, E.C.. Helland, K.N., and van Atta, C.W. (1986). The evolution of grid-generated turbulence in a stably stratified fluid. J. Fluid Mech., 162, 299-338.

Jones, W.P., and Musonge, P. (1988). Closure of the Reynolds-stress and scalar flux equations. Phys. Fluids 31, 3589-3604.

Jongen. J., and Gatski, T.B. (1998). A new approach to characterizing the equilibrium states of the Reynolds stress anisotropy in homogeneous turbulence. Theoret. Comput. Fluid Dimamics, 11. 31-47.

Kaltenbach, H.J., Gerz. T., and Schumann, U. (1994). Large-eddy simulation of homogeneous turbulence and diffusion in stably stratitied shear flow. I. Flaid Mech., 280. 1-40.

Kolmogorov, A.N. (1941). The local structure of turbulence in an incompressible viscous fluid for very large Reynolds numbers. C. R. Acad. Sci., U.S.S.R., 30; 301; Translation: Tumblence. Classic Papers on Statistical Theory (Friedlander. S.K.. and Topper, L., eds.) Interscience. New York 1961.

Launder, B.E. (1976). Heat and mass transport. In Tarbulence - Topies in Applied Physics 12 (Bradshaw, P., ed.) pp. $231-287$. Springer-Verlag. Berlin.

Launder, B.E. (1989). The prediction of forced-field effects on turbulent shear How via second-moment closure. In Adrances in Turbulence - 2. (Fernholz, H.H. and Fiedler. H.E., eds.) pp. 338-358. Springer-Verlag. Berlin.

Launder, B.E. Reece, G., and Rodi, W. (1975). Progress in the development of a Reynolds stress turbulence closure. $J$. Flaid Mech. 68, 537-566.

Lee, M.J., and Reynolds, W.C. (1985). Numerical experiments on the structure of homogeneous turbulence. Report No. TF-24. Department of Mechanical Enginecring, Stanford University. Stanford, CA.

Lumley, J.L., and Khajeh-Nouri. B. (1974). Computational modeling of turbulent transport. Adt: in (re'ophss., 18A. 169-192.

Monin, A.S. (1965). On the symmetry of turbulence in the surface layer of air. Atmos. Ocem. Phys., 1, $45-54$.

Nagano, Y., and Kim. C.A. (1988). Two-equation model for heal transport in wall turbulent shear flows. J. Heat Transfer, 110. $583-589$.

Newman, G.R., Launder, B.E.. and Lumley, J.L. (1981). Modeling of the behavior of homogeneous scalar turbulence. J. Flaid Merh.. $52,609-638$.

Piccirillo, P., and van Atta, C.W. (1997). The evolution of a uniformly sheared thermally stratified turbulent flow. I. Flaid Mech.. 334.

61-86.
Press, W.H., Flannery. B.P., Teukolsky, S.A., and Velterling. W.T. (1986). Numerical Recipes - The Art of Scientific Computing. Cambridge University Press, Cambridge.

Rogallo, R.S., and Moin, P. (1984). Numerical simulation of turbulent flows. Amn, Rev: Fluid Mech., 16, $99-137$.

Rogers. M.M., Moin. P., and Reynolds, W.C. (1986). The structure and modeling of the hydrodynamic and passive scalar tields in homogeneous turbulent shear flows. Report No. TF-25. Department of Mechanical Engineering. Stantord University, Stanford, CA.

Rohr, J.J., Itsweire. E.C. Helland, K.N., and van Atta, C.W. (1988a). An investigation of the growth of turbulence in a uniform mean shear flow. I. Fluid Mech.. 187, 1-33.

Rohr, J.J., Itsweire. E.C. Helland, K.N., and van Alta. C.W. (1988b). Growth and decay of turbulence in a stably stratitied shear flow. I. Fluid Mech., 195. 77-111.

Shabany, Y., and Durbin. P.A. (1997). Explicit algebraic scalar llux approximation. ALAA J., 35, 985-989.

Sirivat, A., and Warhaft, Z. (1983). The effect of a passive cross-stream temperature gradient on the evolution of temperature variance and heat flux in grid turbulence. J. Fluid Mech., 128, 323-346.

So, R.M.C., and Spediale, C.G. (1999). A review of turbulent heat transfer modeling. In Annual Rericw of Heat Transfer. Vol. X (Tien, C.L. ed.). pp., 177-219, Begell House. New York.

So, R.M.C. Zhao, C.Y., and Gatski. T.B. (1999). Predicting buoyant shear flows using anisotropic dissipation rate model. J. Flow Turbulence' Combust., 63. 193-221.

Sommer. T.P., and So, R.M.C. (1995). On the modeling of homogeneous turbulence in a stably stratified flow. Phys. Fluids, 7 ,

$2766-2777$.
Sommer, T.P., So, R.M.C., and Zhang, J. (1997). Modeling nonequilibrium and history effects of homogeneous turbulence in a stably stratified medium. Int. J. Heat Flaid Flow: 18. 29-37.

Speziale, C.G. (1991). Analytical methods for the development of Reynolds stress closures in turbulence. Ann. Rel: Flude Mech., 23. $107-157$.

Speziale, C.G., and Gatski, T.B. (1997). Analysis and modeling of anisotropies in the dissipation rate of turbulence. $I$. Fluid Mech., 344. $155-180$.

Speziale. C.G., and Mac Giolla Mhuiris, N. (1989). On the prediction of equilibrium states in homogeneous turbulence. I. Fluid Mech., 209, 591-615.

Speziale, C.G., Abid, R., and Blaisdell, G.A. (1996). On the consistency of Reynolds stress turbulence cilosures with hydrodynamic stability theory. Phys. Fluids, 8. 781-788.

Speziale, C.G.. Sarkar, S., and Gatski. T.B. (1991). Modelling the pressure-strain correlation of turbulence: an invariant dynamical systems approach. J. Fluid Mech., 227, 245-272. 
Stillinger. D.C.. Helland, K.N.. and van Atta, C.W. (1983). Experiments on the transition of homogeneous turbulence to internal waves in a stratitied fluid. J. Fluid Mech., 131, 91-122.

Tavoularis, S., and Corrsin, S. (1981). Experiments in nearly homogeneous turbulent shear flows with a uniform mean temperature griadient: Part 1. J. Fluid Mech., 104, 311-347.

Tavoularis, S. and Karnik. U. (1989). Further experiments on the evolution of turbulent stress and scales in unilormly sheared turbulence. J. Fluid Mech. 204. 457-478.

Wikstrom, P.M.. Wallin. S., and Johansson. A.V. (2000). Derivation and investigation of a new explicit algebraic model for the passive scalar Ilux. Phys. Fluids, 12, 688-702.

Yoshizawa, A. (1988). Statistical modeling of passive-scalar diffusion in turbulent shear flows. J. Fluid Mech. 195, $541-555$.

Youssel, M.S.. Nagano, Y., and Tagawa. M. (1992). A two-equation heal transfer model for predicting turbulent thermal tields under arbitrary wall thermal conditions. Imt. J. Heat Mass Transfer. 35, 3095-3I04.

Zhao. C.Y. So, R.M.C. Gatski. T.B., and Vimala. P. (2001). A theoretical determination of the equilibrium state parameters of buoyant turbulent shear flows. Phys. Fluids (to be submitted). 